دراسة مختبرية للنحر في الأرضيات الحجرية مؤخر السدود الغاطسة المثلثية من المنظور الأفقي

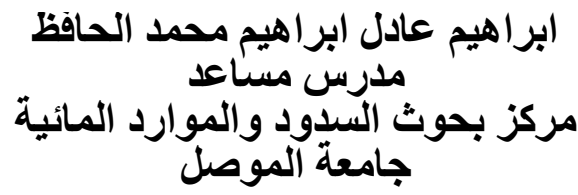

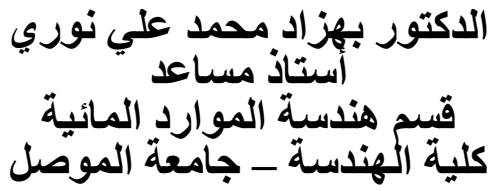

\title{
الخلاصة
}

تم في هذا البحث إجر اء در اسة مختبريـة لظـاهرة النحر في الأرضيات الحجريـة مؤخر

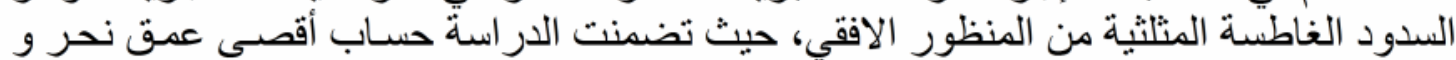

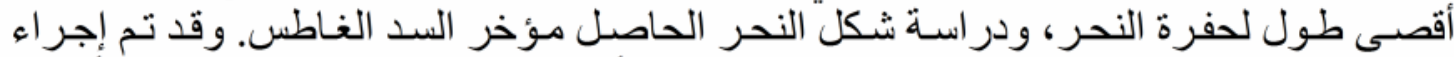



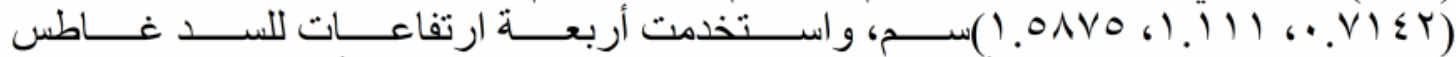



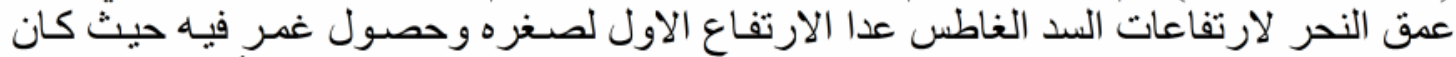

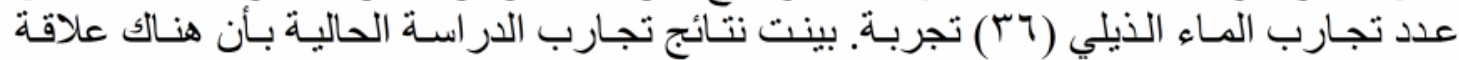

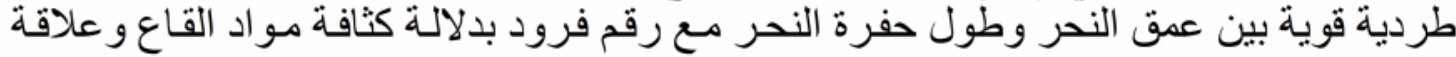



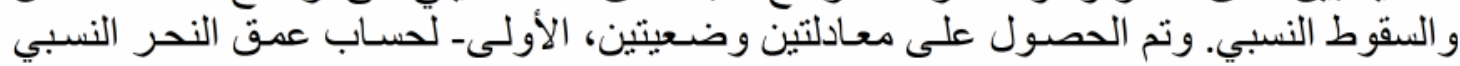

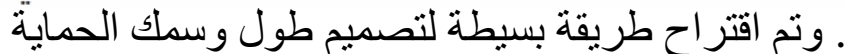

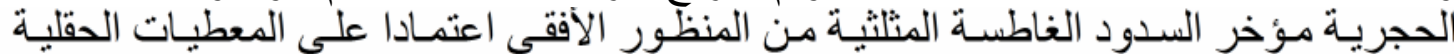
واختبار حجم الحجر المتوفر قرب المبرد الموقع.

\section{Laboratory Study Of Scour In Stone Beds Downstream Triangular Plan Form Weirs}

\author{
Dr. Bahzad M.A.Noori \\ Assistant Professor \\ Dept. Of Water Resources \\ College of Engineering \\ University Of Mosul
}

\author{
Mr. Ibrahim A.I.Al-Hafith \\ Assistant Lecturer \\ Dams and Water Resources \\ Research Center \\ University Of Mosul
}

\begin{abstract}
In this research, the phenomenon of scour downstream triangular plan form weirs on stone beds were studied experiment

ally. The study deals with the measurement of maximum scour depth and the length of scour hole downstream the weir. The shape of scour hole was also studied. One hundred and eight runs were conducted
\end{abstract}


in a laboratory channel. Three sizes of crushed stones of diameters $(0.7142,1.111,1.5875) \mathrm{cm}$ were used. Four weir-heights $(10,15,20,25) \mathrm{cm}$ were tested for all sizes of crushed stones. The effect of tailwater depth on scour depth was studied for all weir-heights except $10 \mathrm{~cm}$ because submergence appeared, while, total number of tailwater depths tested were thirty six. Experimental results of this study showed direct relation between scour depth and scour hole length with the densimetric particle Froude number, but the scour depth and scour hole length had inverse relations with both relative tailwater depth and relative drop. Two empirical equations were obtained; the first for the estimation of relative scour depth and the second for the estimation of relative length of scour hole. A simple design method was presented to obtain the length and thickness of rock protection downstream triangular plan form weirs depending on field data and size of available rocks.

Key words: scour, stone beds, triangular weirs.

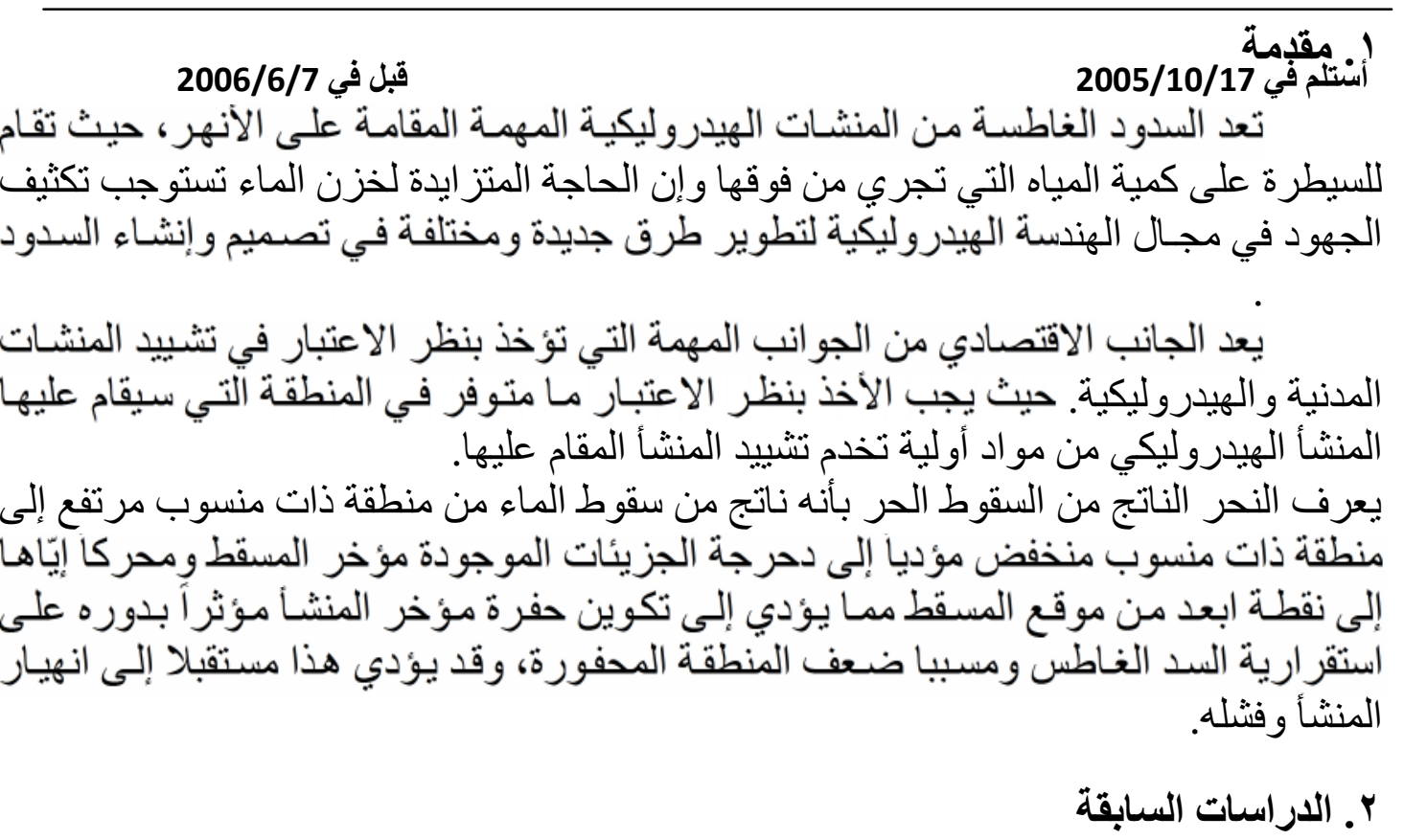

Whittaker and Schleiss (Schoklitsch,1935) (اقترح الباحث سكوكليتش (1984)


الهيدروليكية حيث استتنج المعادلة التالية لحساب عمق النئ درس النحر :

$$
\begin{aligned}
& \mathrm{D}_{\mathrm{s}}=4.75 \mathrm{H}^{0.2} \mathrm{q}^{0.57} / \mathrm{d}_{90}^{0.32}
\end{aligned}
$$

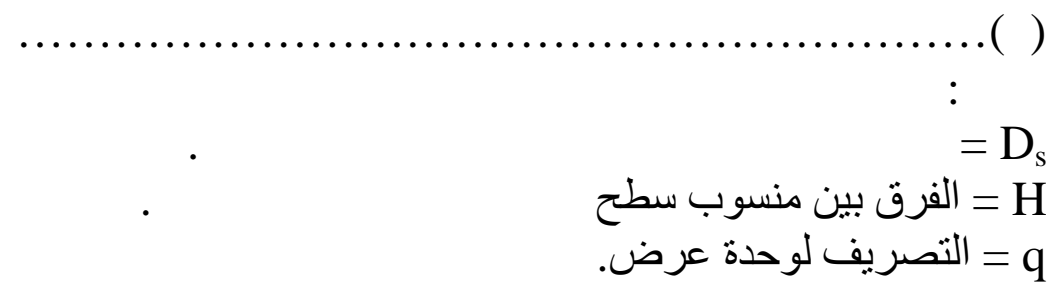


\% $=d_{90}$ Whittaker and Schleiss

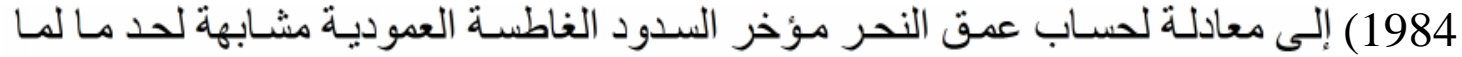
توصل اليه سكو كلينش (Schoklitsch,1935) و هي :

$$
\mathrm{D}_{\mathrm{s}}=3.68 \mathrm{H}^{0.225} \mathrm{q}^{0.54} / \mathrm{dm}^{0.32}
$$


عمق النحر الناتج من جريان الماء فوق وتحت بوابة الكسح وتوصـا الى الى العلاقة التالية لحساب

$$
\text { صيغة جيكر هي : }
$$

$$
\begin{aligned}
\text { وصيغة ايكنبيركر هي } & \text { وt }=\mathrm{D}_{\mathrm{s}}+\mathrm{T}_{\mathrm{w}}=\mathrm{C} \times \Delta \mathrm{h}^{0.5} \times \mathrm{q}^{0.6} / \mathrm{d}_{90}^{0.4}
\end{aligned}
$$

= dt

$=\mathrm{D}_{\mathrm{s}}$

= Tw $=\Delta \mathrm{h}$

C ثابت ويتم حسابه من المعادلة التالية : C

$$
\mathrm{C}=22.8-1 /\left[0.049 \times\left(\mathrm{q}_{\mathrm{u}} / \mathrm{q}_{\mathrm{d}}\right)^{3}-0.0063\left(\mathrm{q}_{\mathrm{u}} / \mathrm{q}_{\mathrm{d}}\right)^{2}-0.029\left(\mathrm{q}_{\mathrm{u}} / \mathrm{q}_{\mathrm{d}}\right)+0.064\right] .
$$

حيث أن التصـريف المـار فوق البو ابـة لوحدة العرض و و و التصـريف المـار تحت البوابـة

Whittaker and Schleiss


1984) النحر مؤخر السدود الغاطسة العمودية للمنشآت الهيدروليكية متوصلا الى العلاقة التالية

$$
\mathrm{D}_{\mathrm{s}}=0.78 \mathrm{H}^{0.35} \mathrm{q}^{0.7} / \mathrm{d}_{90}^{0.4}
$$

وتوصل الباحثان بيساز و تسكوب (Bisaz and Tschopp, 1972) الى معادلة لحساب عمق النحر مؤخر المساقط العمودية:

$$
\mathrm{D}_{\mathrm{s}}=2.7 \mathrm{~h}^{0.25} \mathrm{q}^{0.5}-7.22 \mathrm{~d}_{90}
$$


كمـا درس الباحث بي وآخرون (Pe, et al., 1980) النحر الحاصل مؤخر السدود الغاطسة العمودية وتوصلو الى الى العلاقة التالية لحساب عمق النحر :

$$
\mathrm{D}_{\mathrm{s}}=0.864 \mathrm{~T}^{0.29}(\mathrm{Q} / \mathrm{T})^{0.42} \mathrm{H}^{0.3} / \mathrm{dm}^{0.21}
$$

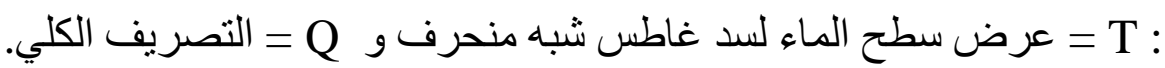





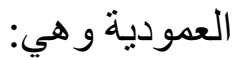

$$
\mathrm{D}_{\mathrm{s}}=\mathrm{kH}^{0.225} \mathrm{q}^{0.54}-\mathrm{T}_{\mathrm{w}}
$$

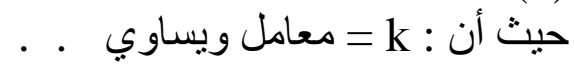

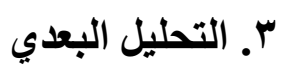

إن ظاهرة النحر الحاصل مؤخر السد الغاطس تعتمد على المتغير ات الاتية:

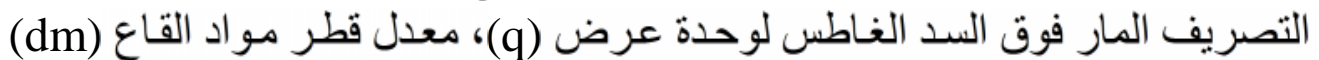

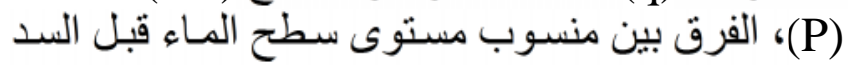

(T) الماء الذيلي (T)

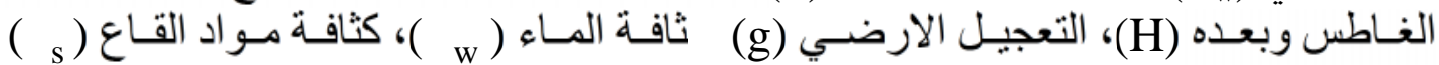
و واللزوجة التحريكية للماء ( ). ويمكن صياغة العلاقة الخاصة بعمق النحر بالثكل الآتي : $D_{s}=f\left(q, d m, T_{w}, P, H, g, \rho_{s}, \rho_{w}, \mu\right)$

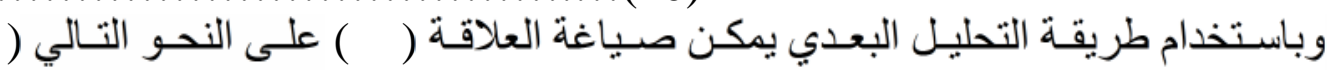
$D_{\mathrm{s}} / \mathrm{dm}_{\mathrm{m}}=\mathrm{f}\left(\mathrm{T}_{\mathrm{w}} / \mathrm{P}, \mathrm{H} / \mathrm{P}, \mathrm{Fr}_{\mathrm{o}}, \mathrm{Re}\right)$

كما يمكن صياغة علاقة لا بعدية لطول حفرة النحر بالثكل التالي : $\mathrm{L}_{\mathrm{s}} / \mathrm{dm}=\mathrm{f}\left(\mathrm{T}_{\mathrm{w}} / \mathrm{P}, \mathrm{H} / \mathrm{P}, \mathrm{Fr}_{\mathrm{o}}, \mathrm{Re}\right)$

$=\mathrm{D}_{\mathrm{s}} / \mathrm{dm}$

$=\mathrm{L}_{\mathrm{s}} / \mathrm{dm}$ يمثل النسبة بين ارتفاع الماء الذيلي وارتفاع السد الغاطس.

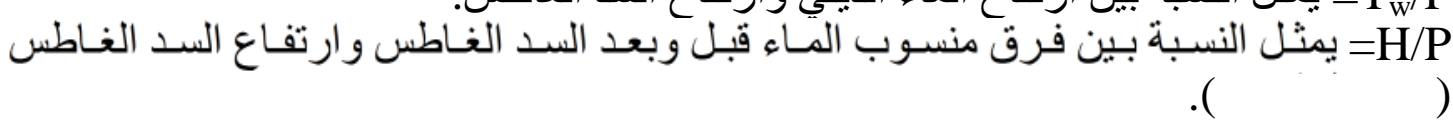

$$
\begin{aligned}
& \cdot\left(q / \sqrt{g\left(\rho_{s} / \rho_{w}\right) d_{m^{3}}}\right) \\
& =\text { Fro } \\
& \text { = رقم رينولدز للجريان ويساوي ( q / Re }
\end{aligned}
$$




$$
\text { = الفرق بين كثافة مو اد القاع وكثافة الماء ( } \rho_{\text {s }} \text { ( ) . }
$$

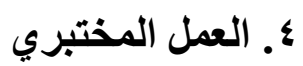

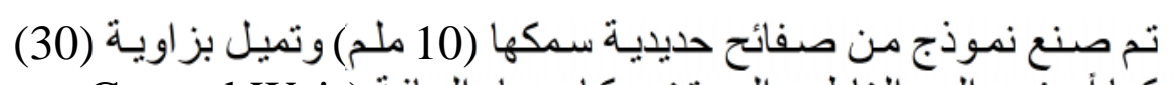

الجدار، كما أن نوع السد الغاطس المستخدم كان حاد الحافة (Sharp Crested Weir)

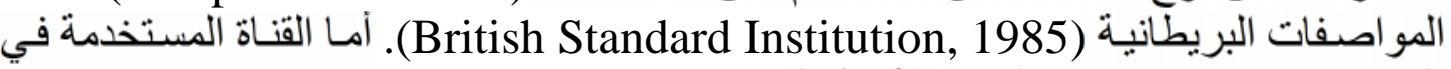
التجـارب فهي مشبيدة من الخرسـانة بطول (24.64 ) )

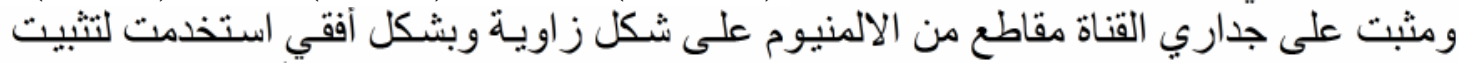

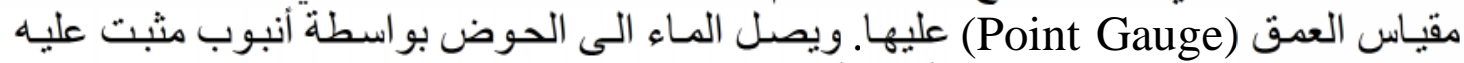

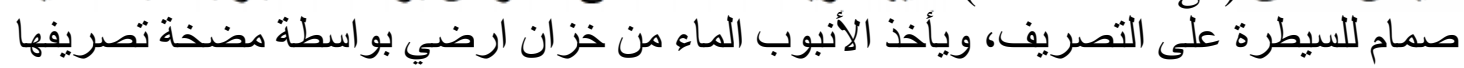

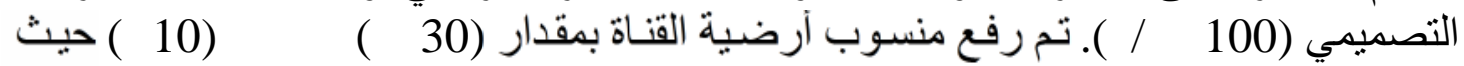

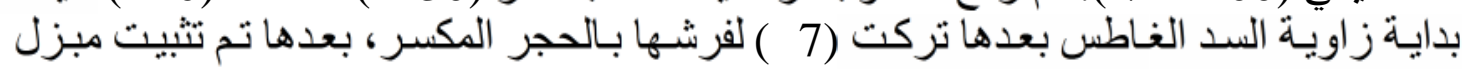

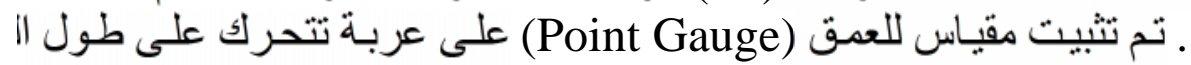

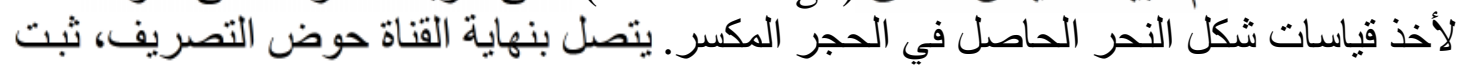

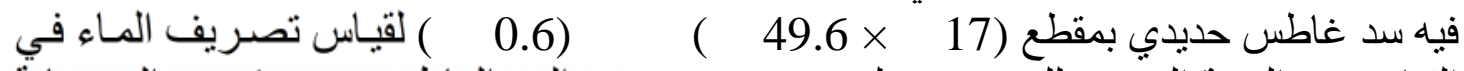

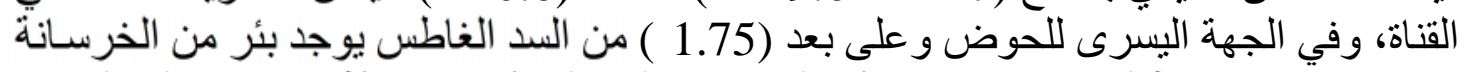

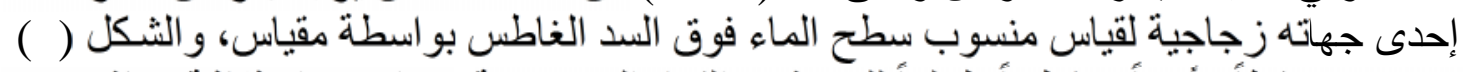

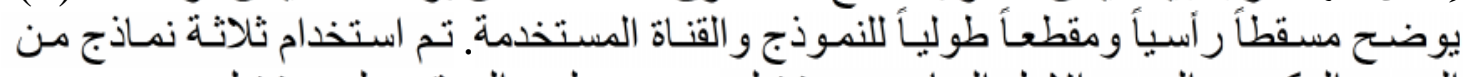

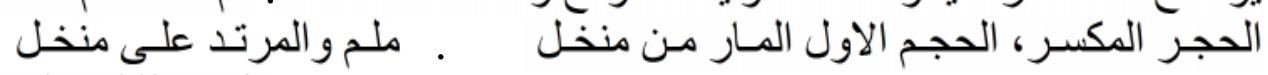

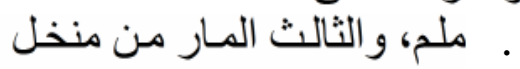

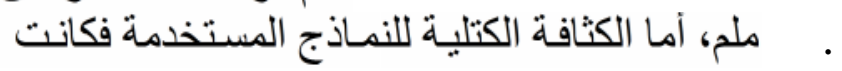

تتلخص خطو ات إجر اء التجارب المختبرية بما يلي: يثبت السد الغاطس أو لا بالارتفاع المطلوب،

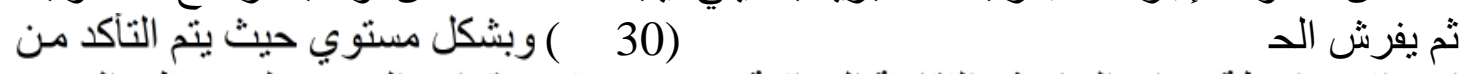

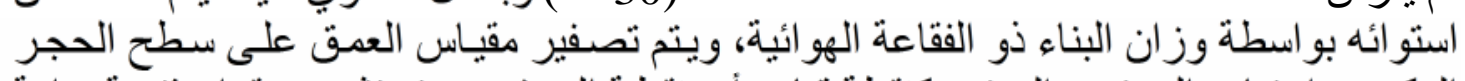

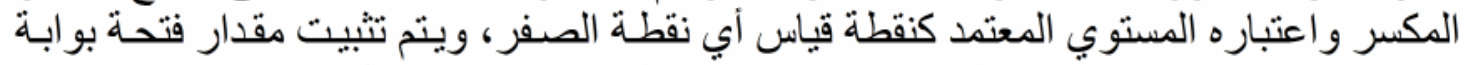

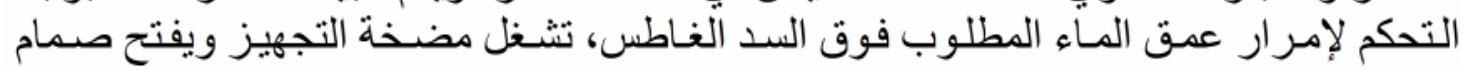

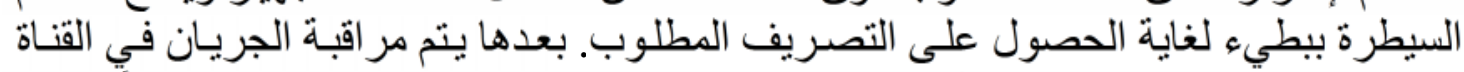



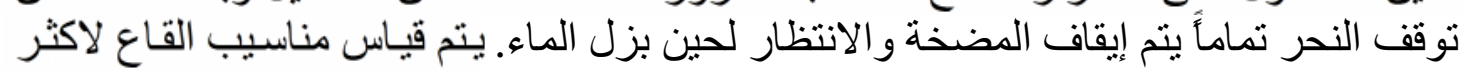





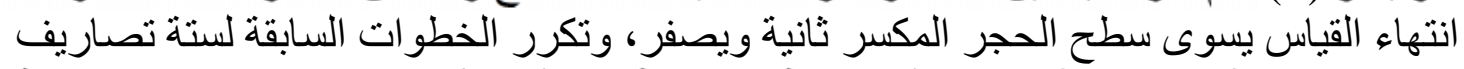

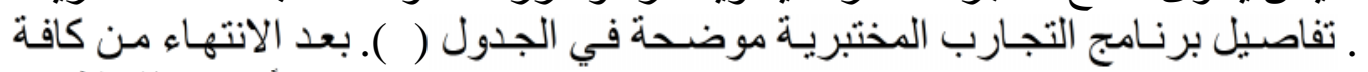

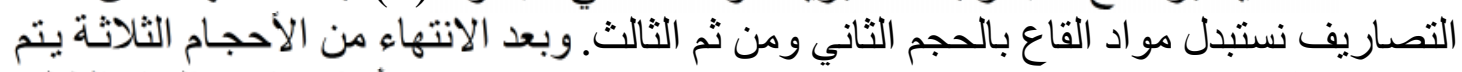

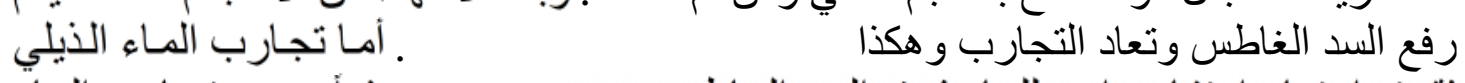


الذيلي بنفس الخطو ات السابقة ولكن برفع منسوب الماء الذباء الذيلي. 


\section{๑. أ. العلاقة بين عمث النحر ورقم فرود بدلالة كثافة مواد القاع}

نظر 1 لأهمية معرفة تأثير تغير Fro على عمق النحر فقد تم تحليل البيانات المختبرية، ووُجدِ بـان


$D_{\mathrm{s}}=\mathrm{a}_{1}+\mathrm{b}_{1} \times$ Fro

( a (a) (13)

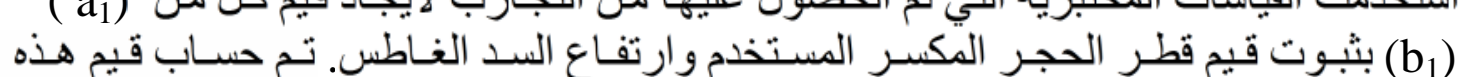

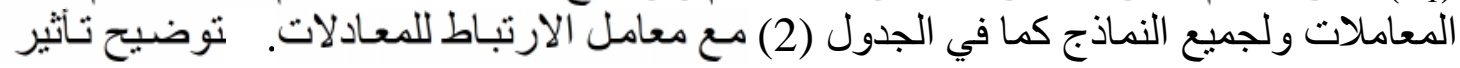
Fro على عمق النحر تم رسم العلاقة بينهما، 


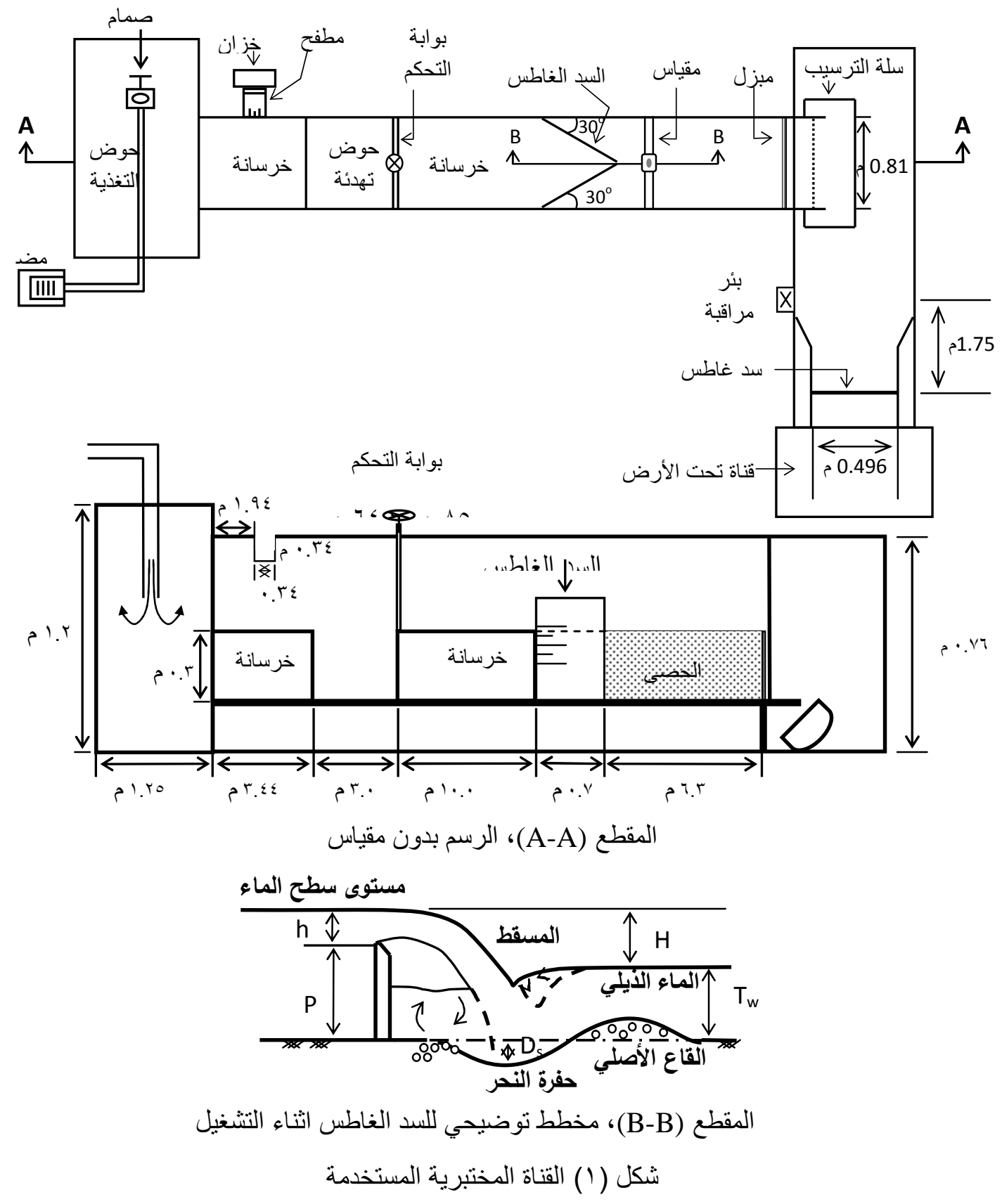

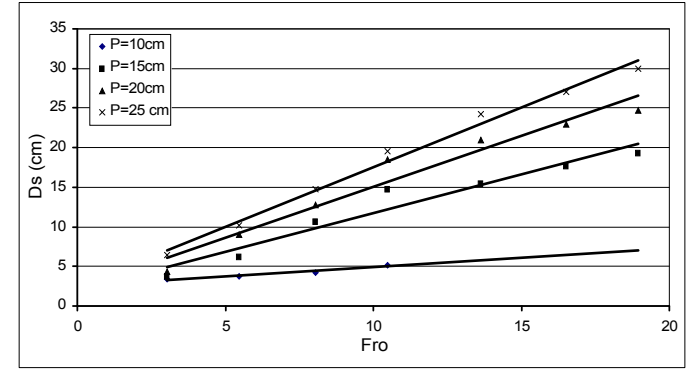

الثكل (ץ): العلاقة بين عمق النحر و Fro لـ ( الـ

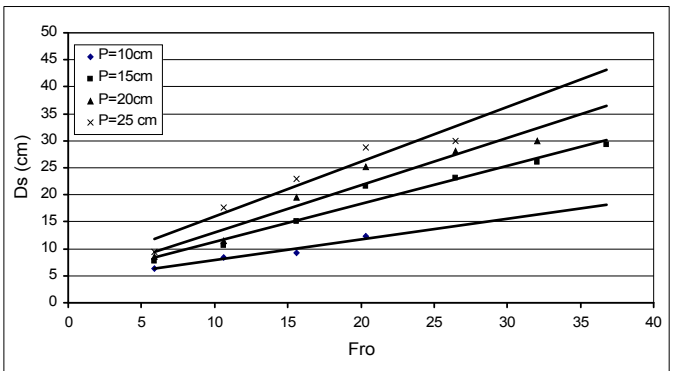


لـ ( ا 

( ) : برنامج التجارب المختبرية

\begin{tabular}{|c|c|c|c|c|c|}
\hline يف لكل & الذيلي ( ) & $(\quad)$ & () & ( ) & \\
\hline - & . - & - & & . & - \\
\hline . - & . - & - & & . & - \\
\hline . - & - & - & & . & 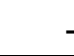 \\
\hline.- & - & - & & . & - \\
\hline 49.54 & - & & & . & - \\
\hline- & - & - & & . & - \\
\hline 49.54 & - & & & . & - \\
\hline $\begin{array}{ll}- \\
-\end{array}$ & - & - & & . & - \\
\hline 49.54 & - & & & . & - \\
\hline- & - & - & & . & - \\
\hline 49.54 & - & & & . & - \\
\hline- & - & - & & . & - \\
\hline 49.54 & - & & & . & - \\
\hline- & - & - & & . & - \\
\hline 49.54 & - & & & . & - \\
\hline- &.- & - & & . & - \\
\hline 49.54 & - & & & . & - \\
\hline- &.- & - & & . & - \\
\hline 49.54 & - . & & & . & - \\
\hline$-\quad$. & - . & - & & . & - \\
\hline 49.54 & - & & & . & - \\
\hline
\end{tabular}
( )
b

\begin{tabular}{|c|c|c|c|c|c|c|c|c|c|}
\hline & \multicolumn{3}{|c|}{$\left.=\mathrm{d}_{\mathrm{m}}\right)$} & \multicolumn{3}{|c|}{$\begin{array}{l}\left.=\mathrm{d}_{\mathrm{m}}\right) \mathrm{B} \\
\quad(\quad 1.111\end{array}$} & \multicolumn{3}{|c|}{$\begin{array}{l}\left.\mathrm{d}_{\mathrm{m}}\right) \mathrm{C} \\
(\quad 1.5875=\end{array}$} \\
\hline $\begin{array}{l}\mathrm{P}(\mathrm{c} \\
\mathrm{m})\end{array}$ & $a_{1}$ & $\mathrm{~b}_{1}$ & $\mathrm{R}$ & $a_{1}$ & $\mathrm{~b}_{1}$ & $\mathrm{R}$ & $\mathrm{a}_{1}$ & $\mathrm{~b}_{1}$ & $\mathrm{R}$ \\
\hline \multirow{2}{*}{10} & 4.07 & 0.38 & 0.97 & 2.57 & 0.23 & 0.96 & 0.41 & 0.59 & 0.99 \\
\hline & 0 & 1 & 57 & 8 & 2 & 85 & 5 & 6 & 20 \\
\hline
\end{tabular}




\begin{tabular}{|c|c|c|c|c|c|c|c|c|c|}
\hline 15 & $\begin{array}{c}4.18 \\
9\end{array}$ & $\begin{array}{c}0.70 \\
3\end{array}$ & $\begin{array}{c}0.98 \\
49\end{array}$ & $\begin{array}{c}1.91 \\
5\end{array}$ & $\begin{array}{c}0.97 \\
8\end{array}$ & $\begin{array}{c}0.97 \\
26\end{array}$ & $\begin{array}{c}0.50 \\
3\end{array}$ & $\begin{array}{c}1.35 \\
2\end{array}$ & $\begin{array}{c}0.97 \\
67\end{array}$ \\
\hline 20 & $\begin{array}{c}4.15 \\
2\end{array}$ & $\begin{array}{c}0.88 \\
0\end{array}$ & $\begin{array}{c}0.97 \\
11\end{array}$ & $\begin{array}{c}2.22 \\
1\end{array}$ & $\begin{array}{c}1.28 \\
4\end{array}$ & $\begin{array}{c}0.97 \\
57\end{array}$ & $\begin{array}{c}0.30 \\
3\end{array}$ & $\begin{array}{c}2.05 \\
0\end{array}$ & $\begin{array}{c}0.98 \\
54\end{array}$ \\
\hline 25 & $\begin{array}{c}5.76 \\
5\end{array}$ & $\begin{array}{c}1.01 \\
4\end{array}$ & $\begin{array}{c}0.96 \\
23\end{array}$ & $\begin{array}{c}2.46 \\
2\end{array}$ & $\begin{array}{c}1.50 \\
7\end{array}$ & $\begin{array}{c}0.99 \\
45\end{array}$ & $\begin{array}{c}3.30 \\
3\end{array}$ & $\begin{array}{c}2.05 \\
6\end{array}$ & $\begin{array}{c}0.99 \\
05\end{array}$ \\
\hline
\end{tabular}

(14)

b

\begin{tabular}{|c|c|c|c|c|c|c|c|c|c|}
\hline & \multicolumn{2}{|c|}{$\mathrm{A}$} & \multicolumn{2}{c|}{$\begin{array}{c}\left.\mathrm{d}_{\mathrm{m}}\right) \mathrm{B} \\
(1.111\end{array}$} & \multicolumn{3}{c|}{$\begin{array}{c}\left.\mathrm{d}_{\mathrm{m}}\right) \mathrm{C} \\
(1.5875=\end{array}$} \\
\hline $\begin{array}{c}\mathrm{P} \\
(\mathrm{cm})\end{array}$ & $\mathrm{a}_{2}$ & $\mathrm{~b}_{2}$ & $\mathrm{R}$ & $\mathrm{a}_{2}$ & $\mathrm{~b}_{2}$ & $\mathrm{R}$ & $\mathrm{a}_{2}$ & $\mathrm{~b}_{2}$ & $\mathrm{R}$ \\
\hline 10 & $\begin{array}{c}9.48 \\
8\end{array}$ & $\begin{array}{c}2.47 \\
8\end{array}$ & $\begin{array}{c}0.98 \\
84\end{array}$ & $\begin{array}{c}12.3 \\
3\end{array}$ & $\begin{array}{c}3.27 \\
9\end{array}$ & $\begin{array}{c}0.97 \\
06\end{array}$ & $\begin{array}{c}10.4 \\
4\end{array}$ & $\begin{array}{c}5.19 \\
2\end{array}$ & $\begin{array}{c}0.97 \\
88\end{array}$ \\
\hline 15 & $\begin{array}{c}6.52 \\
6\end{array}$ & $\begin{array}{c}3.53 \\
6\end{array}$ & $\begin{array}{c}0.94 \\
97\end{array}$ & $\begin{array}{c}18.3 \\
8\end{array}$ & $\begin{array}{c}3.80 \\
0\end{array}$ & $\begin{array}{c}0.98 \\
34\end{array}$ & $\begin{array}{c}14.8 \\
8\end{array}$ & $\begin{array}{c}5.96 \\
4\end{array}$ & $\begin{array}{c}0.99 \\
20\end{array}$ \\
\hline 20 & $\begin{array}{c}17.3 \\
2\end{array}$ & $\begin{array}{c}2.95 \\
7\end{array}$ & $\begin{array}{c}0.99 \\
65\end{array}$ & $\begin{array}{c}19.4 \\
9\end{array}$ & $\begin{array}{c}4.06 \\
5\end{array}$ & $\begin{array}{c}0.98 \\
69\end{array}$ & $\begin{array}{c}15.9 \\
7\end{array}$ & 6.51 & $\begin{array}{c}0.99 \\
40\end{array}$ \\
\hline 25 & 24.0 & 2.68 & 0.99 & 23.4 & 4.06 & 0.98 & 20.6 & 6.49 & 0.98 \\
5 & 0 & 65 & 9 & 5 & 69 & 3 & 7 & 89 \\
\hline
\end{tabular}




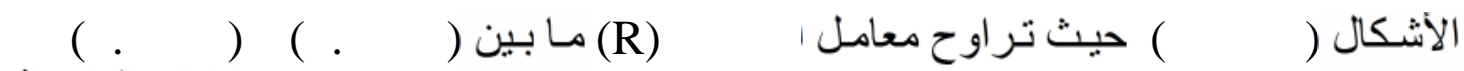

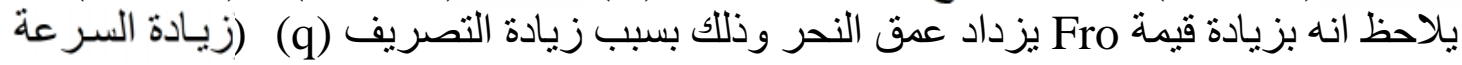

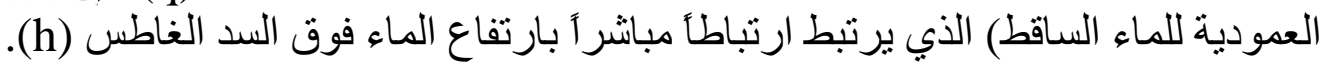

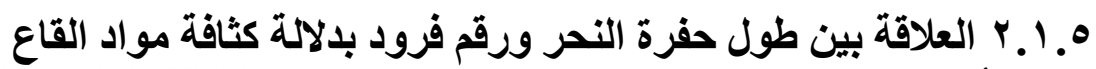

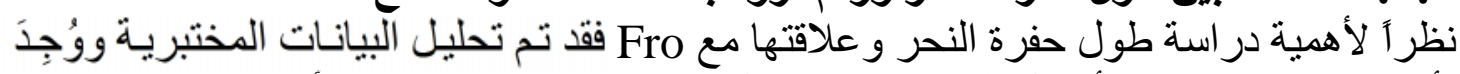
بأن العلاقة الخطية هي أفضل العلاقات بين طئ طول حفرة النحر و Fro وكما ياتٔي: $\mathrm{L}_{\mathrm{s}}=\mathrm{a}_{2}+\mathrm{b} \times$ Fro

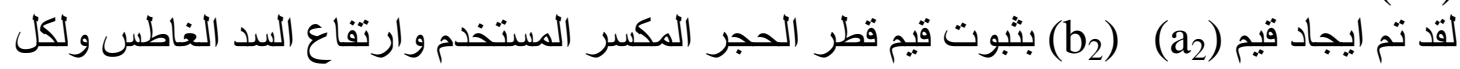

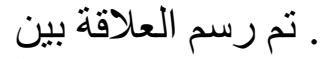

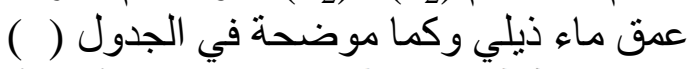
(Fro)

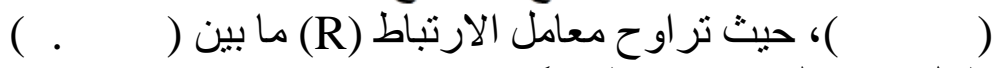

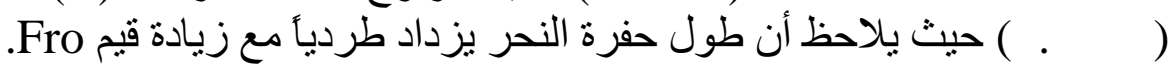

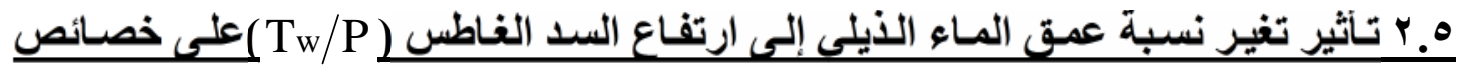

حفرة النحر

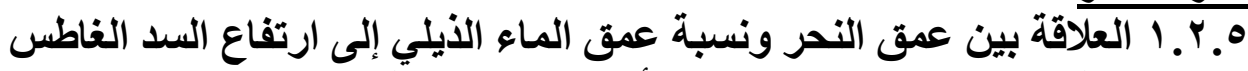



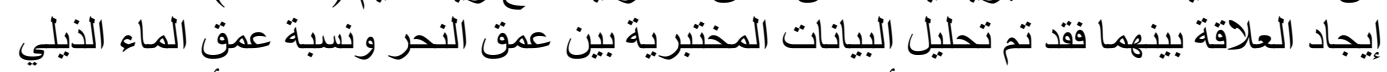
بأن العلاقة الخطية هي افضل بلن العلاقات وكما يأتي:

$\begin{array}{lllllll}\mathrm{D}_{\mathrm{s}} & = & \mathrm{a}_{3} & + & \mathrm{b}_{3} & \times & \mathrm{T}_{\mathrm{w}} / \mathrm{P}\end{array}$

.................................................

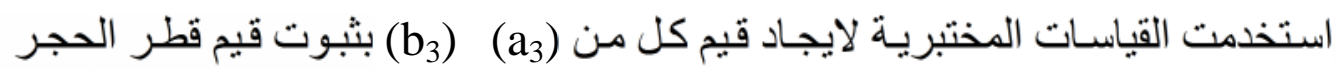

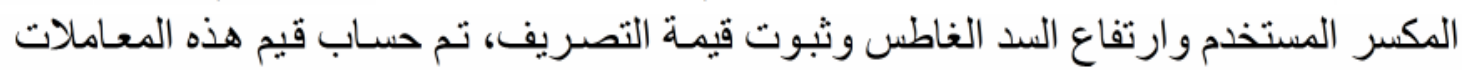

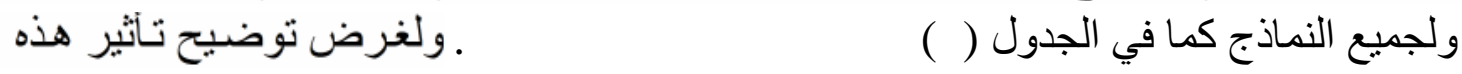



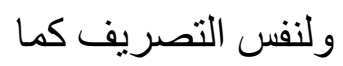





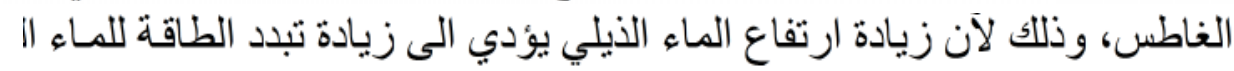

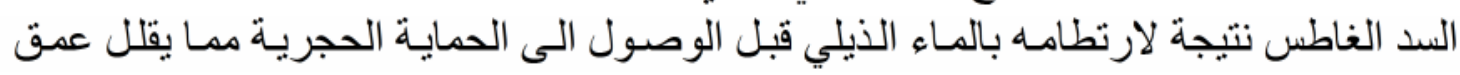





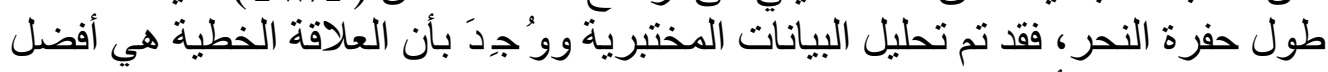




$\begin{array}{lllllll}\mathrm{L}_{\mathrm{s}} & = & \mathrm{a}_{4} & + & \mathrm{b}_{4} & \times & \mathrm{T}_{\mathrm{w}} / \mathrm{P}\end{array}$

استخدمت القياسات المختبرية التي تم الحصول عليها من التجـارب لايجـاد قيم

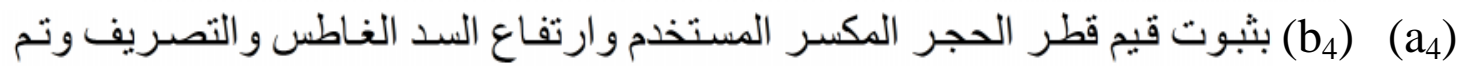
حساب قيم هذه المعاملات ولجميع النماذج كما في الجدول ( ) ) مـع معامل الارنباط للمعـادلات. ولغرض توضيح تأثير هذه النسبة على طول حفرة النحر فقد تم رسم العلاقة بين طول حفرة ر و النسبة بين عمق الماء الذيلي إلى ارتفاع السـ الغـاطس، لارتفاعـات السـ الغـاطس الثاني

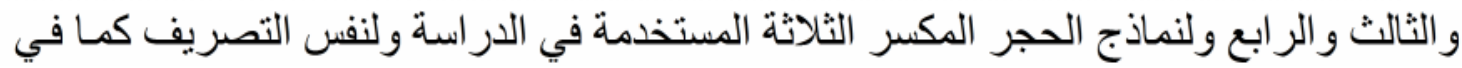

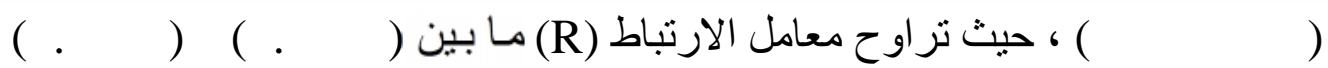






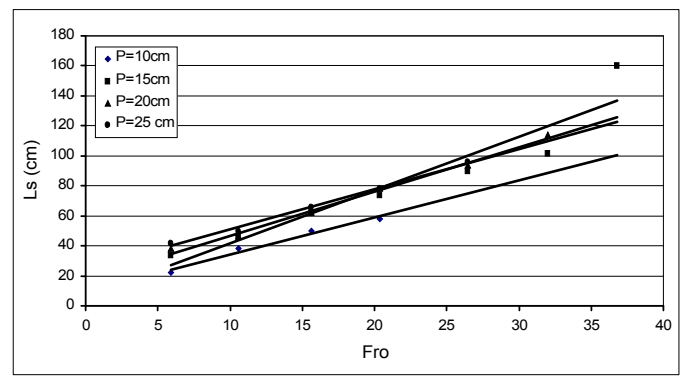

Fro الثكل (0): العلاقة بين طول حفرة النحر و (سم) لـ

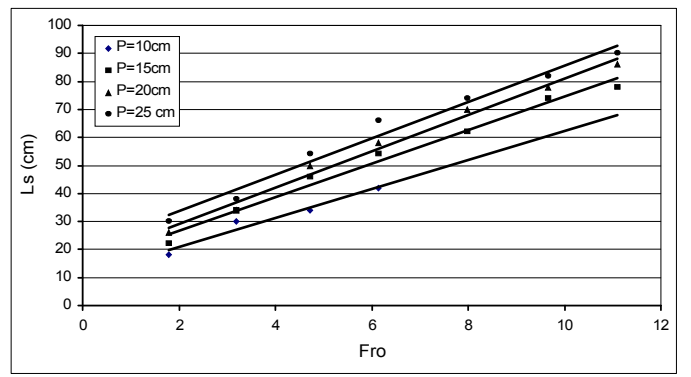









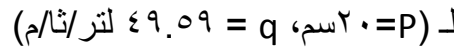

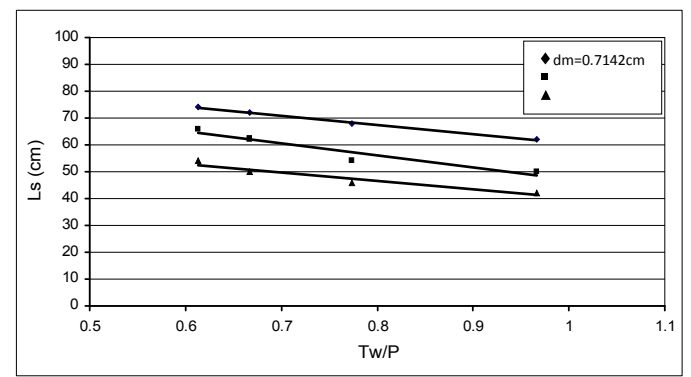



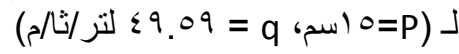

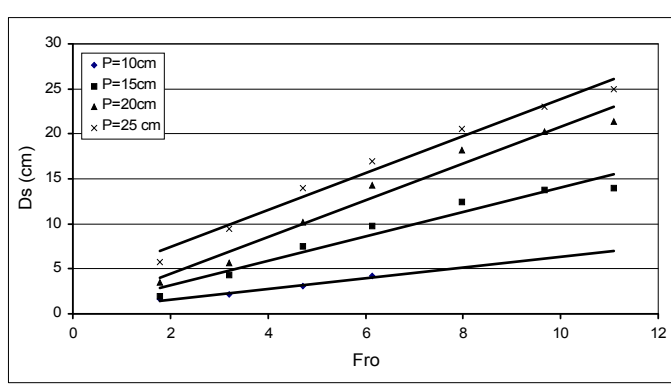

الثكل (ع): العلاقة بين عمق النحر و Fro (س). $0 \wedge \vee 0=\mathrm{dm})\lrcorner$

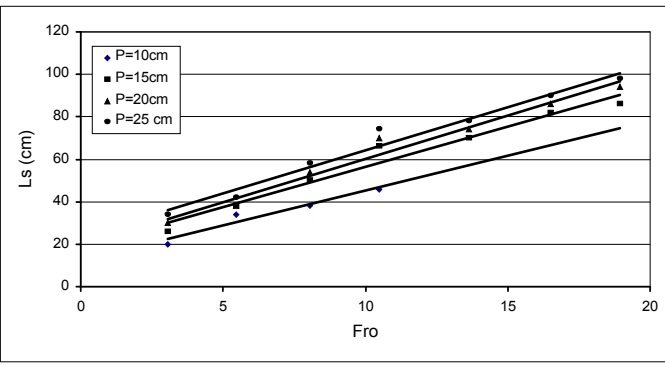

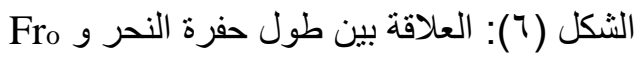
لـ

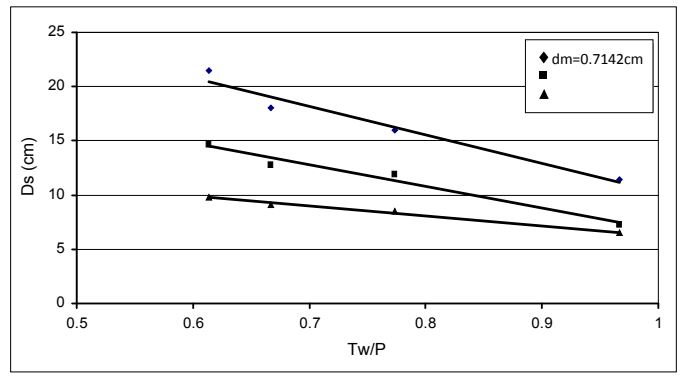

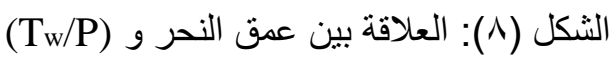

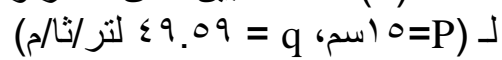

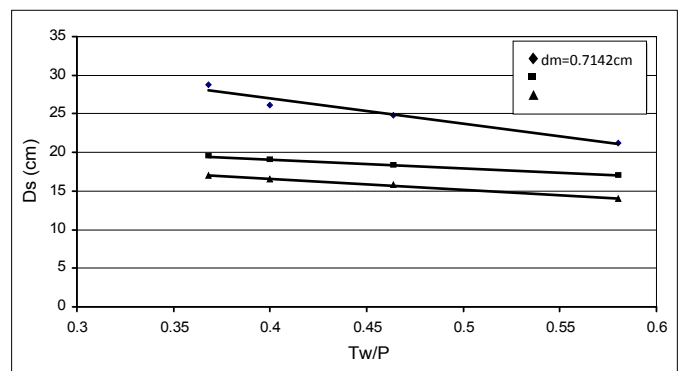

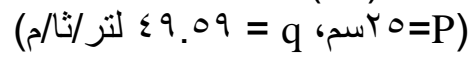


๑. ب تأثير السقوط النسبى (H/P) على خصائص حفرة النحر

هـ ـ. العلاقة بين عمق النحر و السقوط النسبي (H/P)

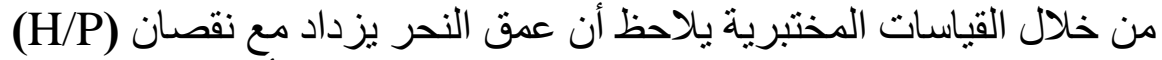

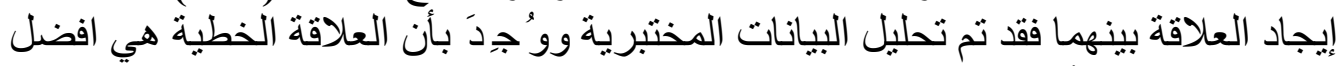

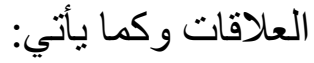

$\begin{array}{lllllll}\mathrm{D}_{\mathrm{s}} & = & \mathrm{a}_{5} & + & \mathrm{b}_{5} & \times & \mathrm{H} / \mathrm{P}\end{array}$

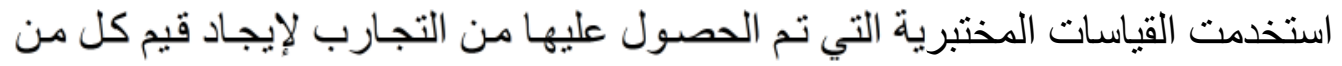

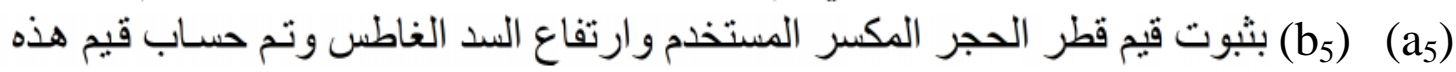

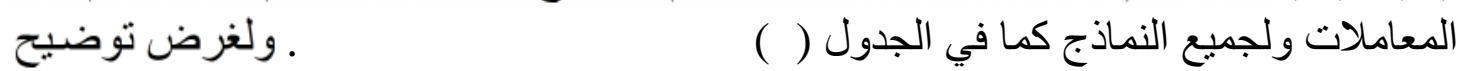

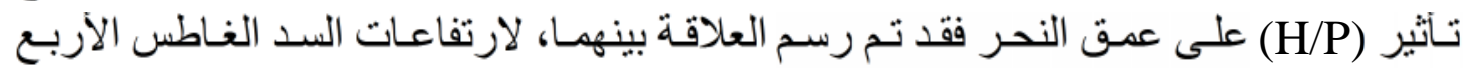

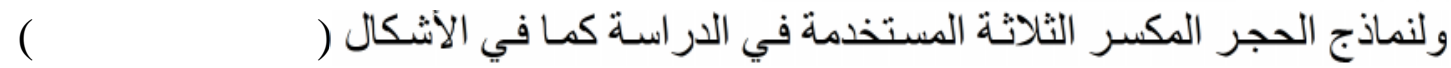

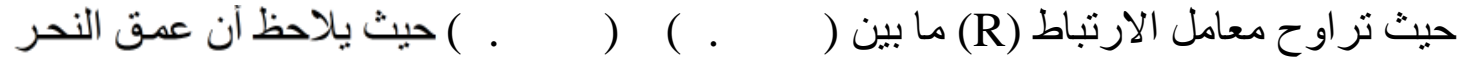

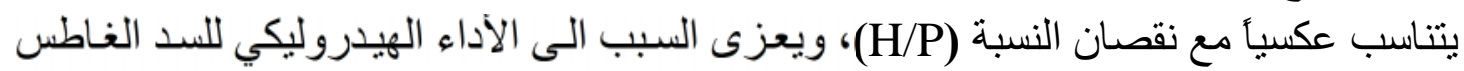

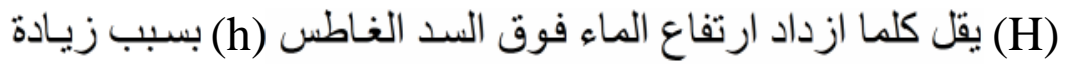

ارتفاع الماء الذيلي (Tw (T) بقيمة اكبر من قيمة (h) وبالتالي يؤدي الى نقصان في قيمة (H).

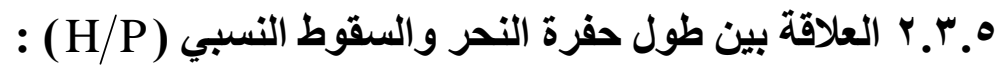

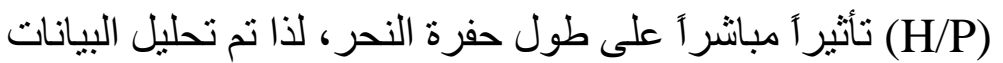

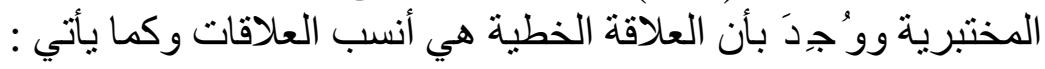

$\mathrm{L}_{\mathrm{s}} \quad \mathrm{a}+\mathrm{b} \quad \times \quad \mathrm{H} / \mathrm{P}$

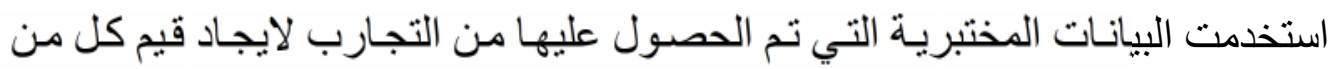

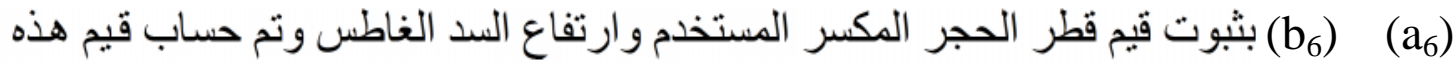

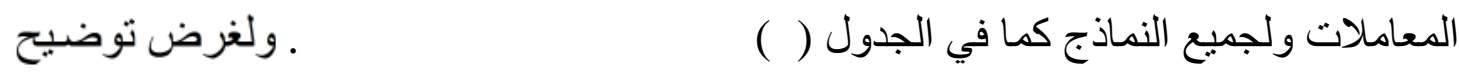

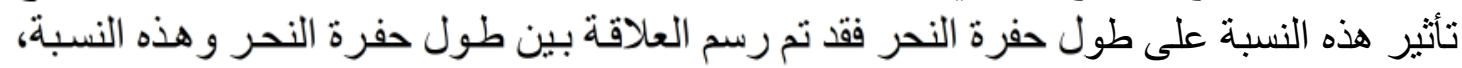

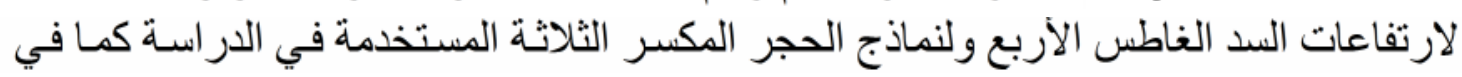

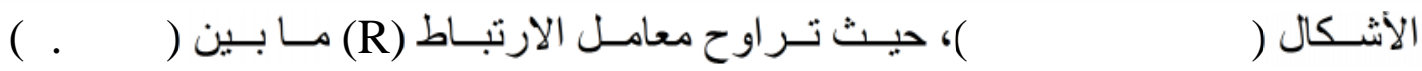






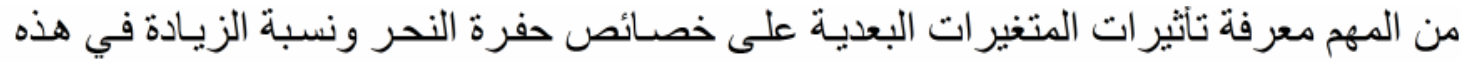

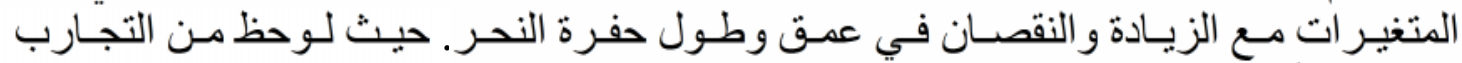

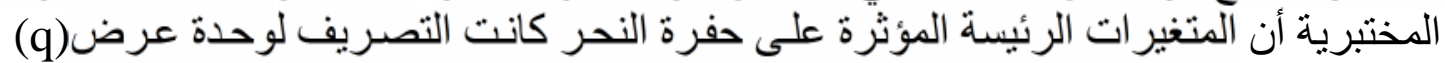



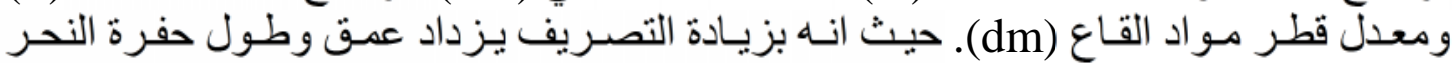

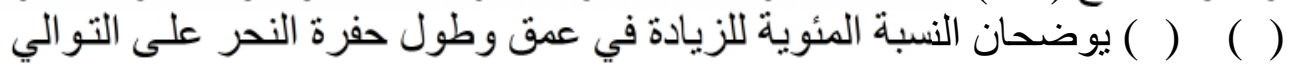

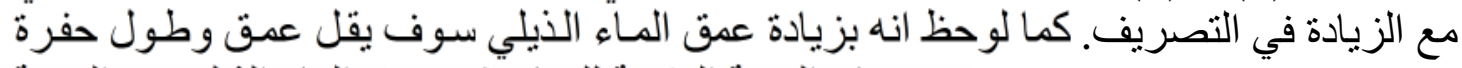

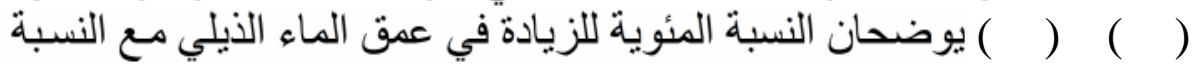

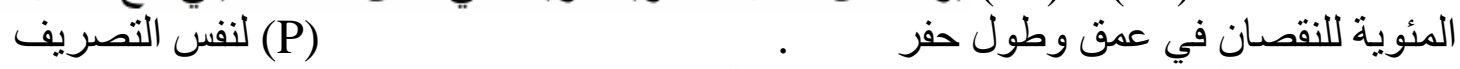



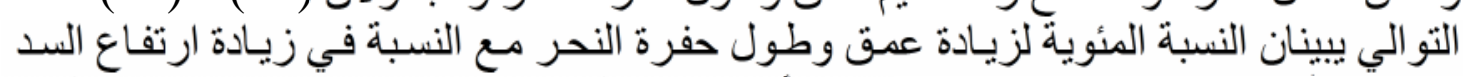



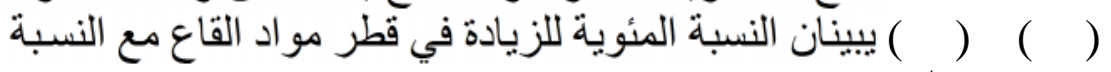
المئوية للنقصان في عمق وامتداد النحر. 



الثكل (13): العلاقة بين طول حفرة النحر و (

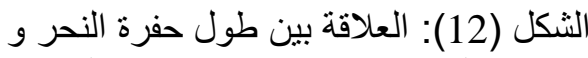
(



الثكل (0 (1): العلاقة بين عمق النحر و(H/P)

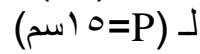

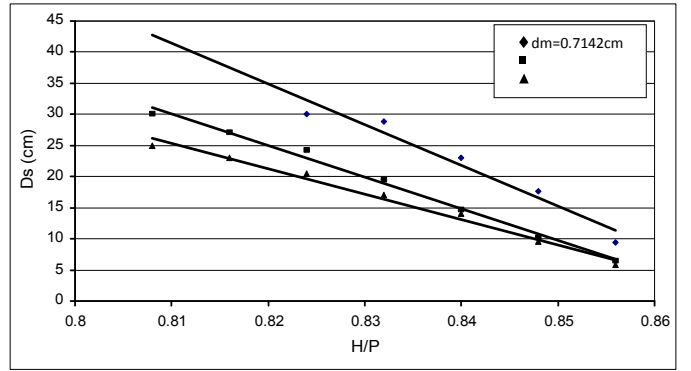

الثكل (V V): العلاقة بين عمق النحر و(H/P)

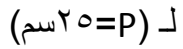

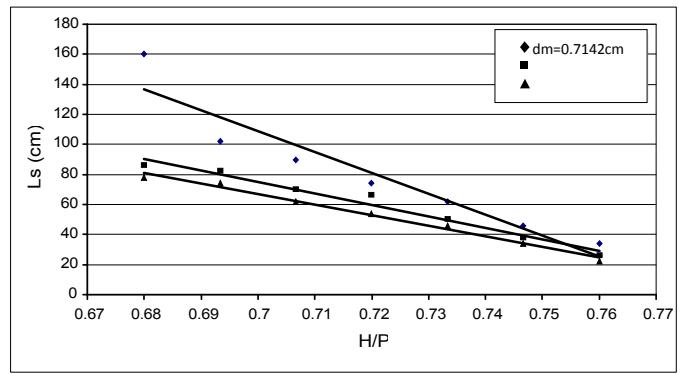

الثكل (ع ()): العلاقة بين عمق النحر و(H/P)

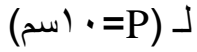

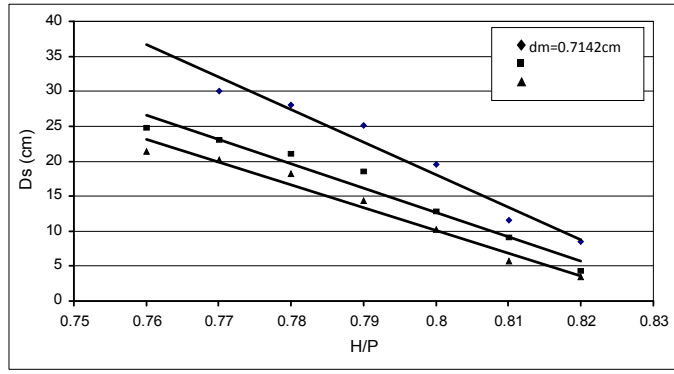

الثكل (7 ( )): العلاقة بين عمق النحر و (H/P) $($ (T)

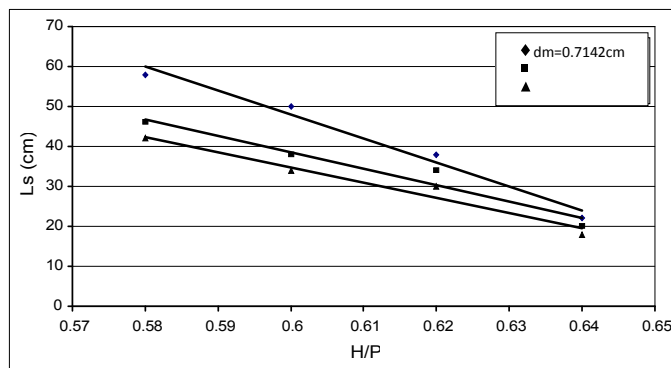

الثكل (18): العلاقة بين طول حفرة النحر و (H/P) الثكل (9 ( ): العلاقة بين طول حفرة النحر و(H/P) لـ 


\begin{tabular}{|c|c|c|c|c|c|c|c|c|c|}
\hline \multicolumn{4}{|c|}{ ( ) } & \multicolumn{4}{|c|}{ b3 a3 قيم المعاملات ( ) } & & \\
\hline & \multicolumn{3}{|c|}{$\left.=\mathrm{d}_{\mathrm{m}}\right)$} & \multicolumn{3}{|c|}{$\begin{array}{l}\left.=\mathrm{d}_{\mathrm{m}}\right) \mathrm{B} \\
(\quad 1.111\end{array}$} & \multicolumn{3}{|c|}{$\begin{array}{l}\left.\mathrm{d}_{\mathrm{m}}\right) \mathrm{C} \\
(\quad 1.5875=\end{array}$} \\
\hline $\begin{array}{c}\mathrm{P} \\
(\mathrm{cm})\end{array}$ & $\mathrm{a}$ & $\mathrm{b}$ & $\mathrm{R}$ & $\mathrm{a}$ & b & $\mathrm{R}$ & $\mathrm{a}$ & b & $\mathrm{R}$ \\
\hline 15 & $\begin{array}{c}36.6 \\
78\end{array}$ & $\begin{array}{c}- \\
26.4 \\
2\end{array}$ & $\begin{array}{c}0.97 \\
81\end{array}$ & $\begin{array}{c}26.6 \\
68\end{array}$ & $\begin{array}{c}19.8 \\
5\end{array}$ & $\begin{array}{c}0.98 \\
61\end{array}$ & $\begin{array}{c}15.3 \\
05\end{array}$ & $\begin{array}{c}9.04 \\
6\end{array}$ & $\begin{array}{c}0.99 \\
40\end{array}$ \\
\hline 20 & $\begin{array}{c}38.6 \\
65\end{array}$ & $\begin{array}{c}- \\
29.9 \\
1\end{array}$ & $\begin{array}{c}0.98 \\
81\end{array}$ & $\begin{array}{c}26.3 \\
76\end{array}$ & $\begin{array}{c}- \\
17.6 \\
2\end{array}$ & $\begin{array}{c}0.99 \\
55\end{array}$ & $\begin{array}{c}19.0 \\
59\end{array}$ & $\begin{array}{c}- \\
11.0 \\
1\end{array}$ & $\begin{array}{c}0.96 \\
48\end{array}$ \\
\hline 25 & $\begin{array}{c}40.1 \\
52\end{array}$ & $\begin{array}{c}- \\
32.9 \\
5\end{array}$ & $\begin{array}{c}0.97 \\
65\end{array}$ & $\begin{array}{c}22.1 \\
62\end{array}$ & $\begin{array}{c}- \\
13.9 \\
9\end{array}$ & $\begin{array}{c}0.99 \\
77\end{array}$ & $\begin{array}{c}22.1 \\
62\end{array}$ & $\begin{array}{c}- \\
13.9 \\
9\end{array}$ & $\begin{array}{c}0.99 \\
77\end{array}$ \\
\hline
\end{tabular}




(17)

b5 ( ) قيم المعاملات

\begin{tabular}{|c|c|c|c|c|c|c|c|c|c|}
\hline & \multicolumn{2}{|c|}{$\mathrm{A}$} & \multicolumn{2}{c|}{$\left.\begin{array}{c}\mathrm{d} \\
\mathrm{m}\end{array}\right) \mathrm{B}$} \\
-
\end{tabular}

(18)

b

\begin{tabular}{|c|c|c|c|c|c|c|c|c|c|}
\hline & & $\begin{array}{l}A \\
\text {. }\end{array}$ & & & $\begin{array}{l}\text { ) } \mathrm{B} \\
1.1\end{array}$ & & & $\begin{array}{l}\mathrm{C} \\
1.587\end{array}$ & \\
\hline $\begin{array}{c}P \\
(\mathrm{~cm})\end{array}$ & $\mathrm{a}_{6}$ & $b_{6}$ & $\mathrm{R}$ & $a_{6}$ & $\mathrm{~b}_{6}$ & $\mathrm{R}$ & $a_{6}$ & $b_{6}$ & $\mathrm{R}$ \\
\hline 10 & 408 & -600 & $\begin{array}{c}0.98 \\
91\end{array}$ & $\begin{array}{c}284 . \\
6\end{array}$ & -410 & $\begin{array}{c}0.97 \\
31\end{array}$ & $\begin{array}{c}262 . \\
8\end{array}$ & -380 & $\begin{array}{c}0.98 \\
12\end{array}$ \\
\hline 15 & $\begin{array}{l}108 \\
0.1\end{array}$ & $\begin{array}{c}- \\
1387 \\
.5\end{array}$ & $\begin{array}{c}0.95 \\
05\end{array}$ & $\begin{array}{c}615 . \\
14\end{array}$ & $\begin{array}{c}- \\
771 . \\
43\end{array}$ & $\begin{array}{c}0.98 \\
79\end{array}$ & 562 & $\begin{array}{c}- \\
707 . \\
14\end{array}$ & $\begin{array}{c}0.99 \\
41\end{array}$ \\
\hline 20 & 129 & $\begin{array}{c}- \\
1542\end{array}$ & 0.99 & 932. & - & 0.99 & 870 & $\begin{array}{c}- \\
1028\end{array}$ & 0.99 \\
\hline
\end{tabular}




\begin{tabular}{|c|c|c|c|c|c|c|c|c|c|}
\hline & 8.6 & .9 & 40 & 71 & 1100 & 08 & & .6 & 54 \\
\hline \multirow{2}{*}{25} & 149 & - & 0.99 & 1211 & - & 0.99 & 1131 & - & 1285 \\
& 4.4 & 1700 & 31 & .7 & 1375 & 08 & $\begin{array}{c}09 \\
.7\end{array}$ & $\begin{array}{c}24 \\
.7\end{array}$ \\
\hline
\end{tabular}

( ) ) نسبة الزيادة في التصريف لوحدة العرض مع نسبة الزيادة في عمق النحر



( ) ) نسبة الزيادة في التصريف لوحدة العرض مع نسبة الزيادة في طول حفرة النحر

\begin{tabular}{|c|c|c|c|}
\hline \multicolumn{2}{|c|}{ نسبة الزيادة في طول حفرة النحر } & \multirow{2}{*}{ التصريف لوحدة في } & \multirow{2}{*}{$\begin{array}{l}\text { نسب الزيادة في } \\
\text { \%h }\end{array}$} \\
\hline الزيادة & ( ) للزيادة) & & \\
\hline \multirow{6}{*}{40} & $(-)$ & 80 & \\
\hline & $(-)$ & & \\
\hline & $(-)$ & & \\
\hline & $(-\quad)$ & & \\
\hline & $(-\quad)$ & & \\
\hline & $(\quad-\quad)$ & & \\
\hline
\end{tabular}




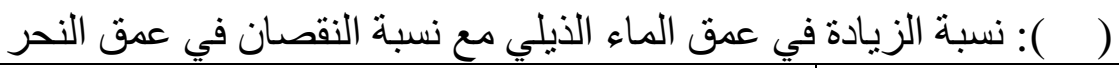



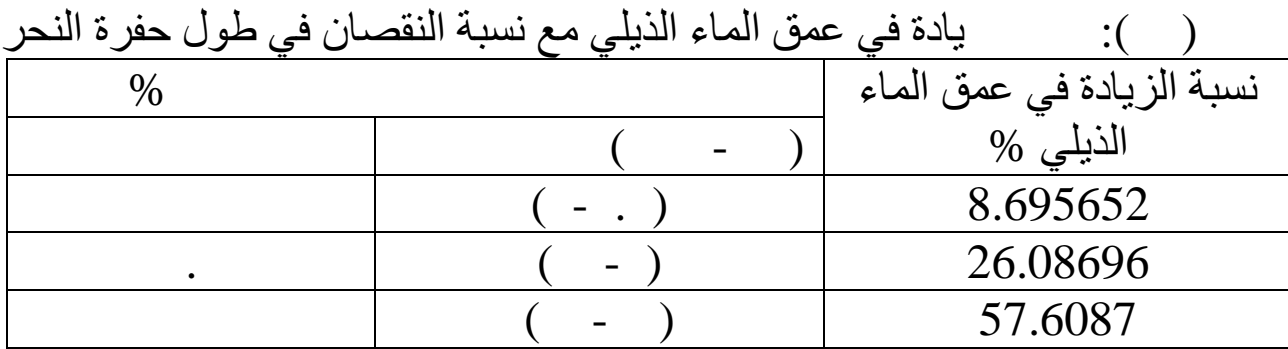

( ) ( ) نسبة الزيادة في ارتفاع السد الغاطس مع نسبة الزيادة في عمق النحر



( ) ) نسبة الزيادة في ارتفاع السد الغاطس مع نسبة الزيادة في طول حفرة النحر

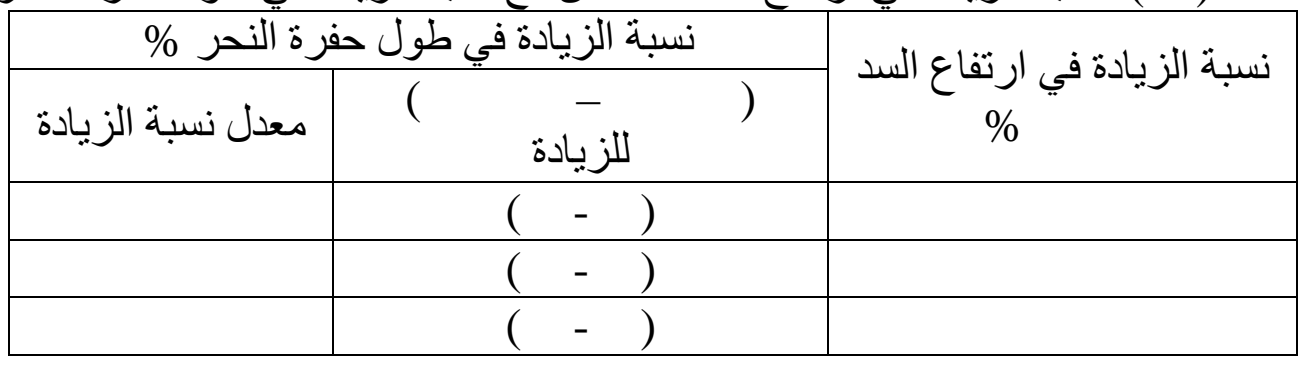

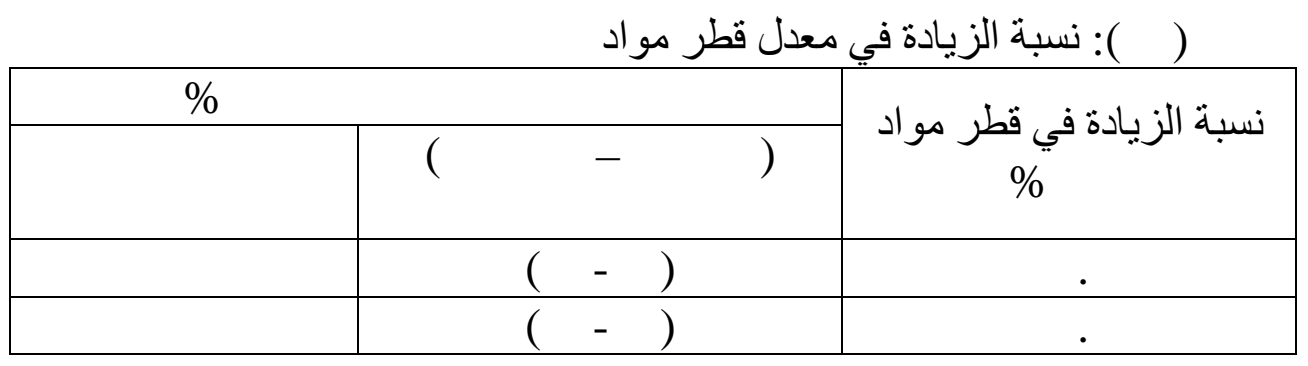

( ) : نسبة الزيادة في معدل قطر مو اد القاع مع نسبة النقصان في طول ح

\begin{tabular}{|c|c|c|}
\hline \multicolumn{2}{|l|}{$\%$} & \multirow{2}{*}{ نسبة الزيادة في قطر } \\
\hline ( & - & \\
\hline
\end{tabular}




\begin{tabular}{|l|c|c|}
\hline & $(-)$ &. \\
\hline & $(-)$ &. \\
\hline
\end{tabular}

V. تغيير شكل عمق النحر عرضياً



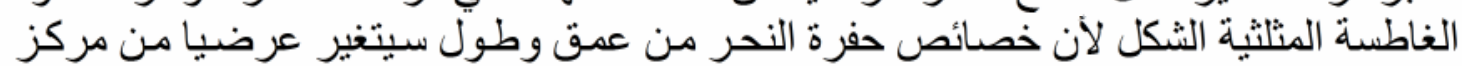

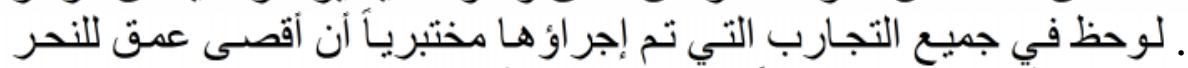

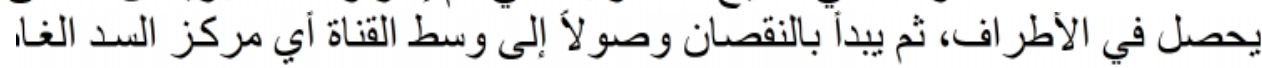

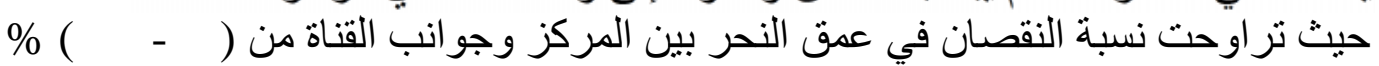

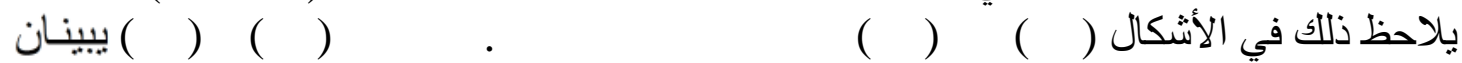

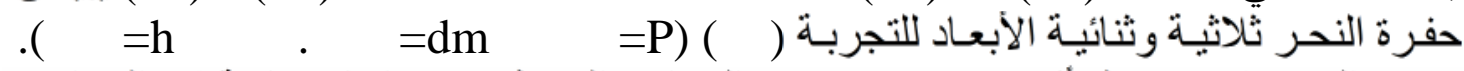

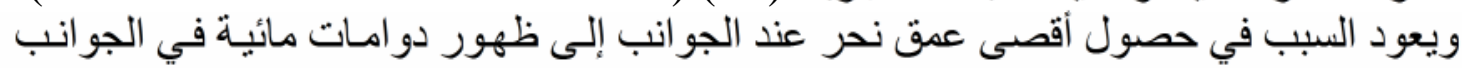





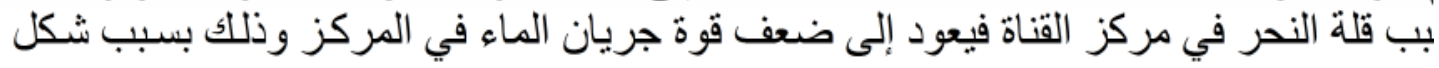

^. استنباط علاقة وضعية لحساب عمق النحر مؤخر السد الغاطس المثلثي الثكل

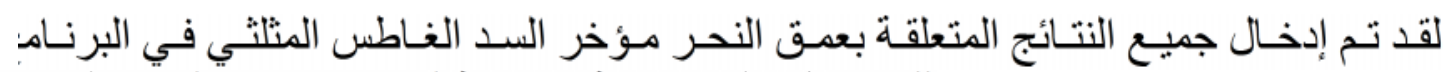

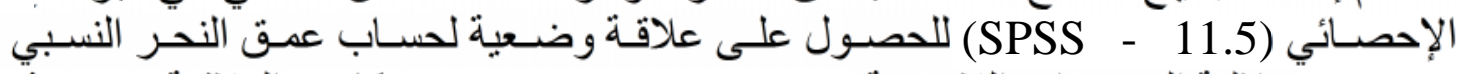


المتغير ات كالآتي: $\frac{\mathrm{Ds}}{\mathrm{dm}}=0.6255 \times\left(\mathrm{Fr}_{\mathrm{o}}\right)^{1.144} \times(\mathrm{H} / \mathrm{P})^{0.296} /\left(\mathrm{T}_{\mathrm{w}} / \mathrm{P}\right)^{0.71618} \ldots \ldots \ldots \ldots \ldots \ldots \ldots \ldots \ldots . .(\mathrm{c})$


9. استنباط علاقة وضعية لحساب طول حفرة النحر مؤخر السد الغاطس المثلثي : تم إذخال جميع البيانات المتعلقة بطول حفرة الندر مؤخر السدود الغاطسـة المثلثيـة في البرنـامج (SPSS - 11.5) بدلالة المتغيرات (Tw/P) (H/P) (Fro) وكما يلي : 


$$
\begin{aligned}
& \frac{\mathrm{L}_{\mathrm{s}}}{\mathrm{dm}_{\mathrm{m}}}=4.0268 \times\left(\mathrm{Fr}_{\mathrm{o}}\right)^{0.9825} /\left(\left(\mathrm{T}_{\mathrm{w}} / \mathrm{P}\right)^{0.3401} \times(\mathrm{H} / \mathrm{P})^{0.3666}\right) \\
& \text { ويمكن تبسيط المعادلة ( ) يساوي ( ) للحصول على المعادلة الآتية:- } \\
& \frac{\mathrm{L}_{\mathrm{S}}}{\mathrm{dm}}=4.0268 \times\left(\mathrm{Fro}_{\mathrm{o}}\right)^{0.9825} \times(\mathrm{P})^{0.7067} /\left(\left(\mathrm{T}_{\mathrm{w}}\right)^{0.3401} \times(\mathrm{H})^{0.3666}\right) \ldots \ldots \ldots \ldots \ldots . .(\quad)
\end{aligned}
$$

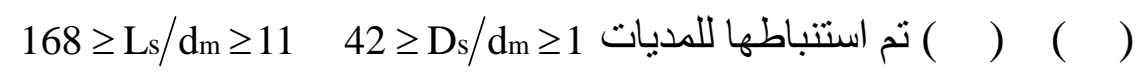

$$
\begin{aligned}
& .0 .86 \geq \mathrm{H} / \mathrm{P} \geq 0.58 \quad 0.92 \geq \mathrm{T}_{\mathrm{w}} / \mathrm{P} \geq 0.22 \quad 36.77 \geq \mathrm{Fr}_{\mathrm{o}} \geq 1.78 \\
& \text {. }
\end{aligned}
$$

أن الإجراءات التصميمية تعتمد في الاساس على النتائج التي تم الحصـول علبها مسبقا

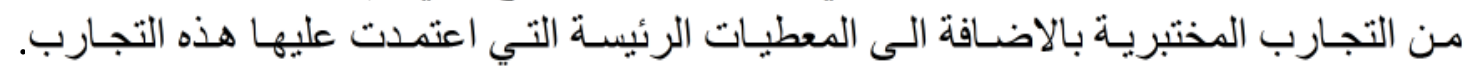

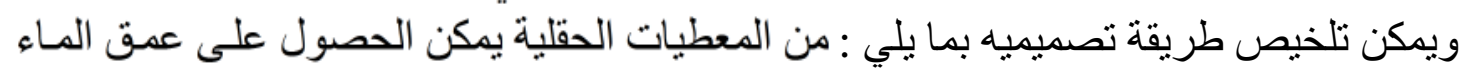



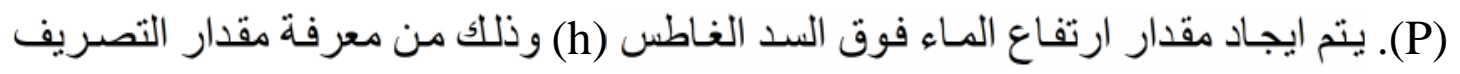

\begin{tabular}{|c|c|c|c|}
\hline$q$ & $=$ & 2.6171 & $x$ \\
\hline
\end{tabular}



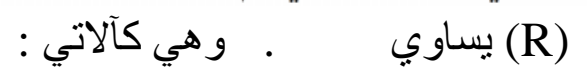




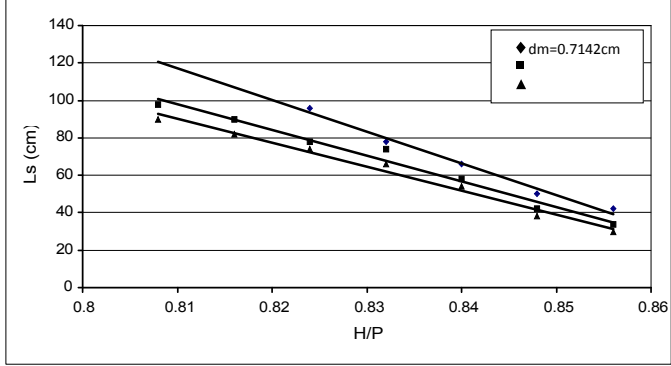

الثكل ( آY): العلاقة بين طول حفرة النحر و) و لـ (Y)





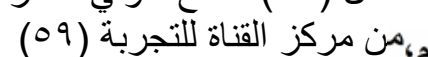

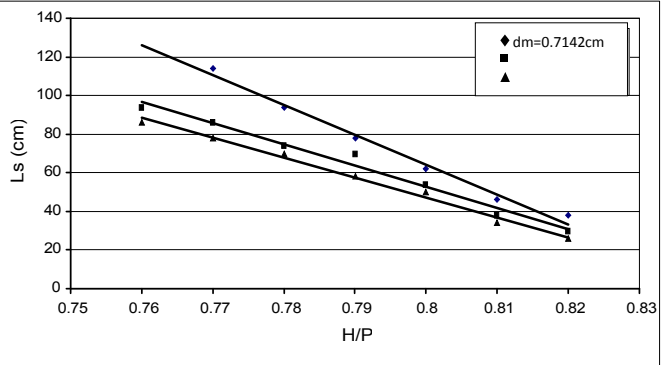

الثكل (· r): العلاقة بين طول حفرة النحر

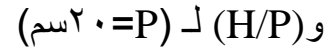

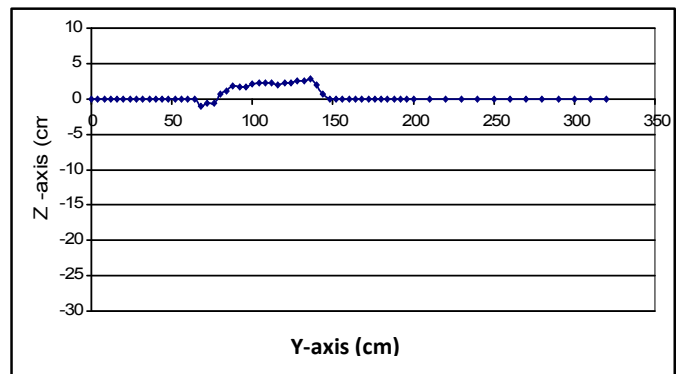

شكل (Yr) مقطع طولي للنحر عند مركز القناة


(0)

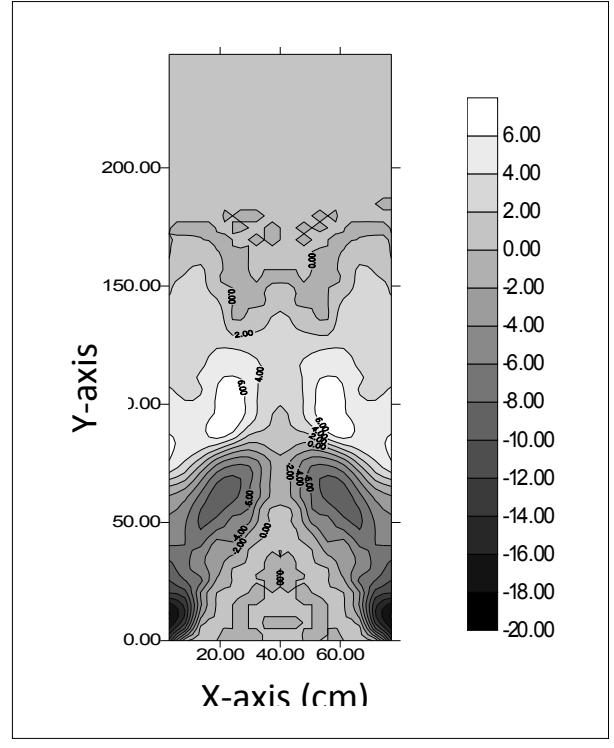

شكل (TT) مقطع ثنائي الأبعاد لشكل النحر الحاصل للتجربة (ب9 الابعاد (ه)
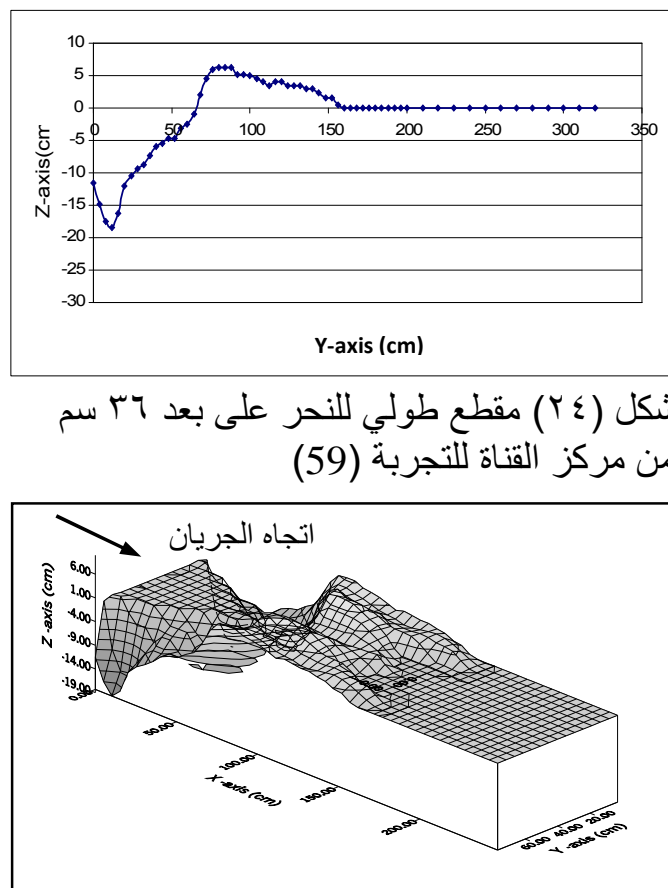

شكل (25) مقطع ثلاثي الابعاد لثكل النحر الحاصل للتجربة (109) 



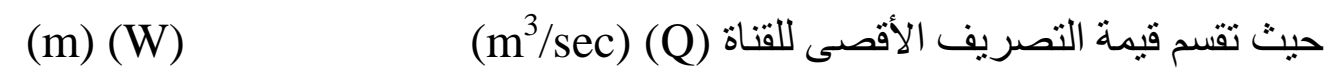
على أقصى تصريف لوحدة عرض القناة (q2/sec) (q2)، التي بدور ها تدخل في المعادلة ( )

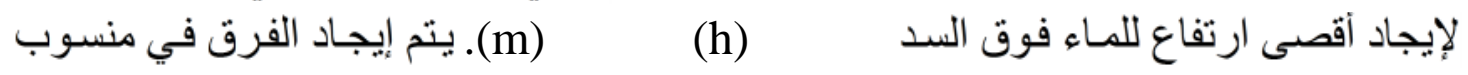
أي مقدار السقوط من المعادلة التالية : (H)

$\begin{array}{lllllllllllllllllllll}\mathrm{H} & \mathrm{h} & + & \mathrm{P} & - & \mathrm{Tw}\end{array}$

يتم بعدها اختيار قيمة لمعدل قطر مواد القاع (dm) حسب توفر ها. بعدها نجد عمق النحر

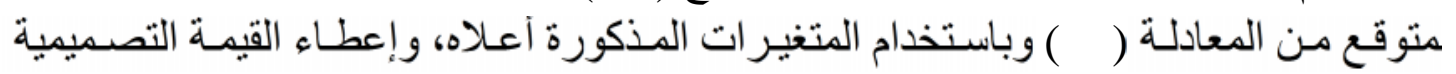


يساوي 1.5 (Smith, 1985). و لإيجاد طول حفرة النحر الحاصلة مؤخر السد الغاطس تستخدم (2) وللحصول على القيمة التصميمية لطول الحماية المطلوبة التي تساوي مقدار طول

.1 .5

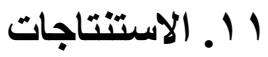

. تم الحصول على علاقة طردية قوية بين عمث النحر (Ds) ورقم فرود بدلالة كثافة مواد



تصـف تـأثير Fro • ( اتضح بأن هنالك علاقة طردية قوية بين طول حفرة النحر (Ls) و ورقم فرود بدلالة كثافة

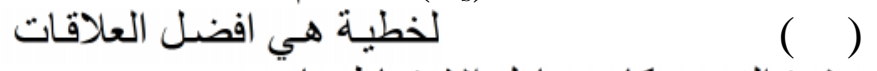

(Fro)

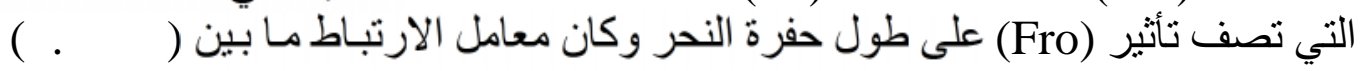

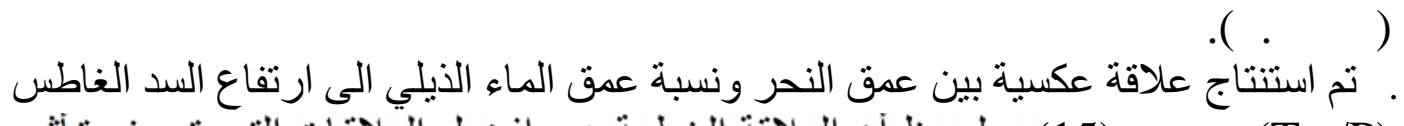

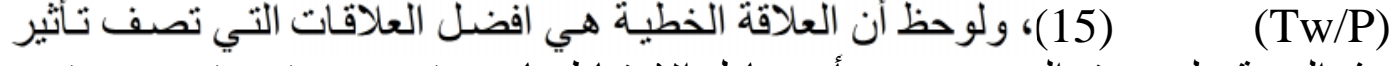



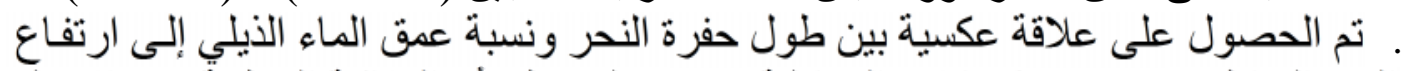

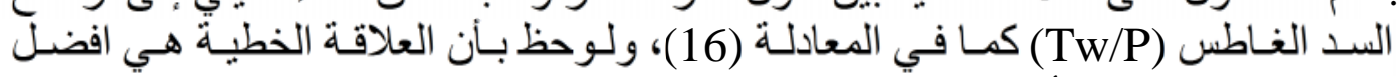



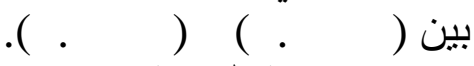
• تمت در اسة العلاقة بين عمق النحر و السقوط النسبي (H/P) وتمت ملاحظة علاقة عكسية

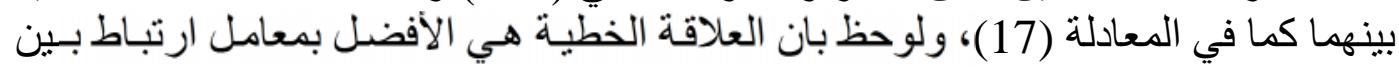

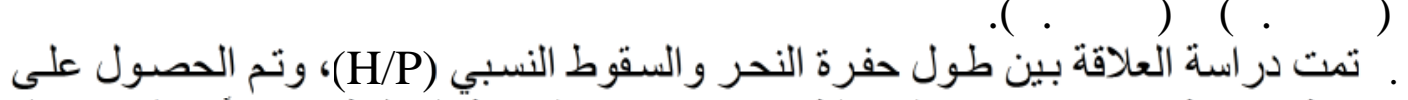

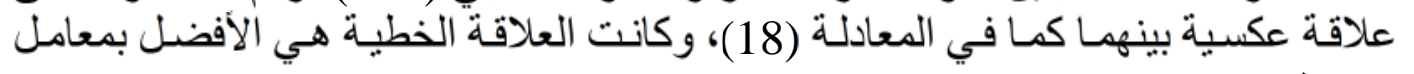






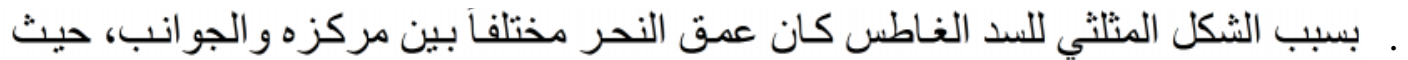

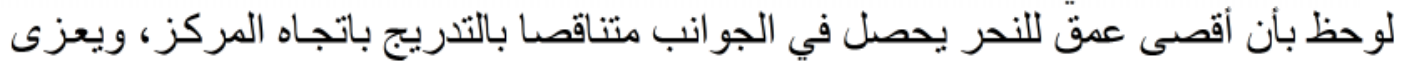

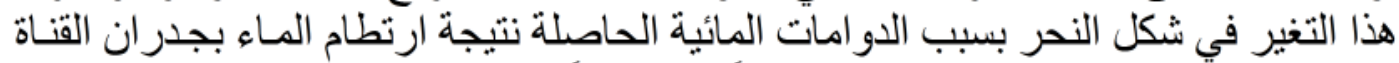

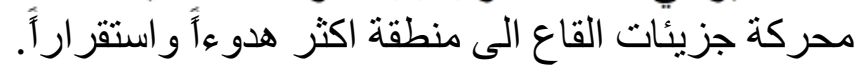

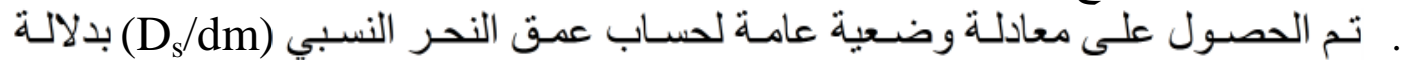

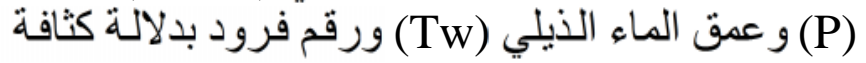

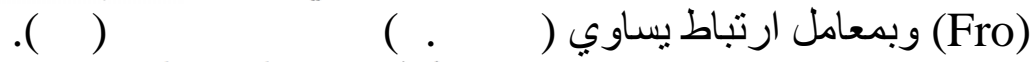



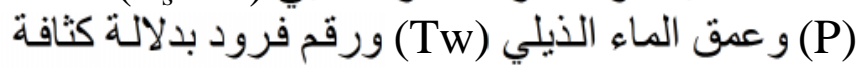

$$
\text { (Fro) }
$$


الحقلية للقناة واختيار حجم الحجر المناسب المتوفر.

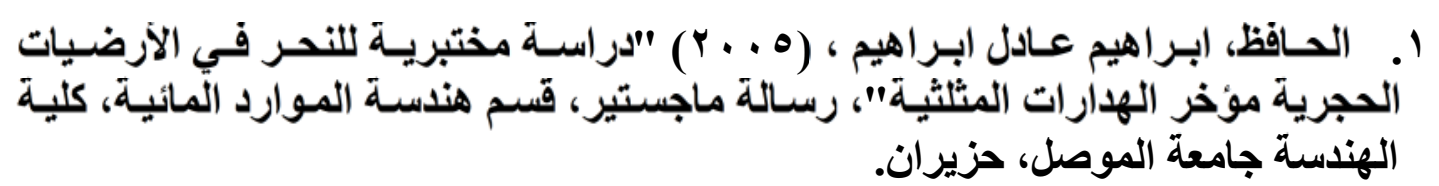

2. Bisaz, E., and Tschopp, J. (1972), "Profundidad de Erosion al Pie de un Vertedero Para la Aplicacion de Correccion de Arroyos en Quebradas Empinadas", Proceedings of the $5^{\text {th }}$ Congreso Latinoamericano de Hidraulica (IAHR), Lima, Peru, 23-28, October, (in Spanish). PP. 447456.

3. British Standard Institution. (1985), "Methods of Measurement of Liquid Flow in Open Channel”, London, England, BS3680, (PartA).

4. Eggenberger, W. (1943), "Die Kolkbildung bein einen uberstromen und beider kombination uber stromen - unterstromen" , Dissertation, ETH Zurich.

5. Jaeger, C. (1939), “Ueber die Achnlichkeit bei Flussaulichen Modeellrer suchen", W.U.W. 34, No. 23-24, 269.

6. Pe, J.A., Achinte, J.R., and Jegat, H.J. (1980), "Estudio Experimental de Caida de Seccion Trapecial", Proceeding of the $9^{\text {th }}$ Congress Latinoamericano de Hidraulica, Vol. 1, Merida, Venezuela, June 30-July 4, (in Spanish). PP. 447-456.

7. Smith, C.D. (1985), "Hydraulic Structures", University of Saskatchewan Printing Services, Saskatoon, Canada.

8. U.S.B.R. (1995), "Stream Stability at Highway Structures" Hydraulic Engineering Circular No.20, Federal Highway Administration.

9. Whittaker, J., and Schleiss, A. (1984), "Scour Related to Energy Dissipaters for High Head Structures", Mitteilungen der Versuchsanstalt 
fur Wasserbau, Hydrologie und Glaziologie, No. 73, an der Eidgenossischen Technischen Hochscule Zurich. 